\title{
High Throughput Quantum Dot Based LEDs
}

\author{
P. Amini, M. Dolatyari, G. Rostami and A. Rostami \\ Additional information is available at the end of the chapter \\ http://dx.doi.org/10.5772/59092
}

\section{Introduction}

Today lighting and energy savings is an important part of life and traditional methods of lighting are inefficient, while the light emitting devices (LEDs) can be used in technologies applications for saving energy [1].LEDs produce light using a fundamentally different principle than those used by incandescent, neon, fluorescent, or high-intensity discharge (HID) lamps. Traditional light sources produce light by heating a filament to incandescence and this is inefficient but LEDs emit light from a small semiconducting chip when a current is applied. So controlling equipment and techniques is one of the most cost-effective and significant opportunities for reduce energy waste and improve output light quality. Researches have also provided new structures to improve the efficiency of LED devices. Conjugated polymers offer many advantages as materials for use in light-emitting diodes [2]. Because of the advantages of quantum dots and their application in optoelectronic devices such as LEDs, researches fabricated quantum dot light emitting diode with improvement efficiency; which was replaced with traditional light sources. Quantum dot light emitting diodes use in energy-efficient, highcolor-quality thin-film display and solid-state lighting applications [3].

In 1907, H. J. Round found the effect of electroluminescence, so the notion of light emitting diode was introduced [4]. III-V materials were discovered in the 1950s, and by using of these materials the first p-n junctions GaAs LED, with epitaxial growth was made [5, 6]. In 1962 GaAsP LED with visible red light was realized by Holonyak [7]. In 1992, the first blue LED based on GaN with efficiency of $1 \%$ was introduced by Akasaki et al [8], also green LEDs could be made using GaInN with improvement efficiencies up to $10 \%$ [9]. The possibility of color displays with blue, red, and green LEDs was successfully realized [10]. Different LEDs can have different characteristics, so they have different application in related technologies. According to the importance of LEDs in new technologies, in recent years there are so motivations by doing a lot of researches to improve the quality of LEDs, so recently they introduced quantum dot based light emitting diodes (QD-LEDs) $[3,11]$. According to im- 
provement of QD-LEDs the efficiency of this kind of LEDs has increased from $0.01 \%$ to $18 \%$, in 1994 [3]. Nano crystals also known as quantum dots (QDs), are nano-sized semiconductor particles, by the synthetic method, the QDs can be classified into epitaxial and colloidal QDs. Colloidal QDs are chemically synthesized and consist of small inorganic semiconductor core (1-10 $\mathrm{nm}$ in diameter) and a wider-band gap inorganic semiconductor shell, and a coating of organic passivation ligands [3]. Original QDs contain a core; however, the optical properties of QDs can be improved by coating higher band gap materials or passivation of the surface of the core [12]. Colloidal QDs exhibit some advantages such as larger excitonic interaction between the electrons and holes, stronger carrier confinement, high quantum yield, narrow emission spectrum. Therefor colloidal QDs have the potential to change the way that electronic devices, including solar cells and LEDs, are manufactured [13, 14]. QDs exhibit quantum confinement effect, because of their radius which is smaller than the characteristic Bohr exciton radius, so the carriers in all three directions confined and the density of states will change. According to quantum confinement effect, the optical properties such as absorption and emission can be changed [12]. Band gap is tunable by controlling the size of the QDs during the synthesis process and cheap solution processability of them makes colloidal quantum-dot a promising candidate for optoelectronic devices $[3,15]$. The color emitted from QDs and the wavelength of emission, depends on the size of the QDs. According to changing in the size of QDs, the emission wavelength can be changed, so the color of light emission can be changed, also QDs have the high photo-luminescence efficiency; therefore these advantages make colloidal QDs candidates for making quantum-dot light emitting diodes [3, 12]. Figure 1 shows the size changing of QDs and band gap of them.

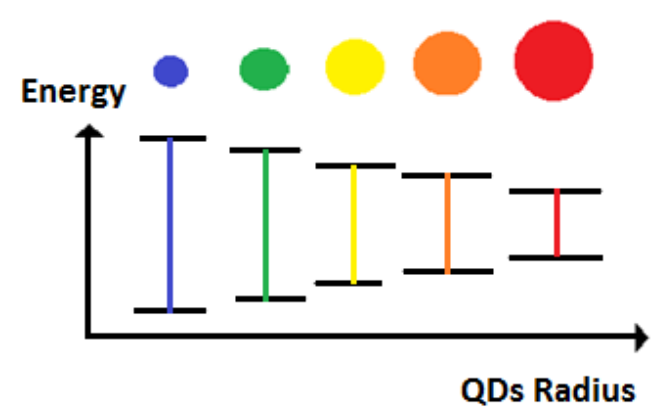

Figure 1. Increasing of energy gap by decreasing of the size of the QDs

QD-LEDs are multilayer structures, consist of hole transport layer (HTL), electron transport layer (ETL) and QD layer as an emissive layer. In this type of LEDs, Indium tin oxide (ITO) (as an anode) and $\mathrm{Al}$ (as a cathode) are used as usual electrodes [3, 16]. At first QD-LEDs presented in the form of hybrid organic/inorganic light emitting diodes by Colvin et al [16]. For the hybrid diodes, the QDs were fabricated from inorganic semiconductors and the transport layers from organic semiconductors. This kind of LEDs was later improved by Coe-Sullivan et al [16]. 
However organic transport layers have stability problems and these layers are sensitive to air, because of these problems the efficiency of hybrid organic/inorganic LEDs decreases [3, 16]. Therefore, inorganic semiconductor transport layers were developed and used in QD-LEDs. Using of inorganic charge transport layers lead to device stability in air [16]. Nevertheless the advantages of QD-LEDs and using them in optoelectronic devices like thin film displays, which cause the improvement in color saturation in this kind of displays, there are some problems limit the applicability of QD LEDs which can be listed as follows:

1. Efficient non-radiative Forster resonant energy transfer (FRET) of excitons within the inhomogeneous size distribution of QDs to non-luminescent sites, where they have nonradiative recombine, cause self-quenching phenomenon.

2. Quenching in photoluminescence (PL) of QDs by the surrounding conductive metal oxides because of carrier imbalance (due to a large hole or electron injection barrier between the $\mathrm{p}$ or $\mathrm{n}$ type metal oxides and the QDs) [3, 17].

Using of inorganic charge-transport materials is desirable because they can facilitate charge carrier injection and transport to the QD layer and also improve the charge confinement in the QD luminescent layer. As a result it is expected that HTL and ETL make the LEDs less susceptible to the problems [18]. Nowadays $\mathrm{NiO}$ and $\mathrm{ZnO}$ are common materials to use as charge transport layers, which $\mathrm{NiO}$ is a p-type material as a $\mathrm{HTL}$ and $\mathrm{ZnO}$ is a n-type material as an ETL $[16,18]$. Engineering the defect energy levels in the structure of $p$ and $n$ type metal oxides can improve their charge transport properties. These defects levels can be engineered using synthesis methods and also by doping of different atoms in the structure of materials. These defect levels act as a radiative recombination center, therefore photon production probability will be increased [19]. The other way to increasing intensity of emitted light of LEDs is Forster resonance energy transfer (FRET). In this way inorganic materials act as core and organic molecules as capping materials. FRET is an energy transfer between these organic and inorganic materials, which one of them is as electron donor and the other one is as electron acceptor [20].

\section{Physics and theory of LEDs and QD-LEDs}

LEDs are pn-junction diodes which have many applications in displays and lighting. These LEDs made from III-V semiconductors. Figure 2 shows pn-junction and energy level of it, which indicate pn-junction under zero bias and forward bias. The junction between p-type and n-type is non-conductive, because of moving of electrons by diffusion from the n-type region into the p-type region and combine with the acceptors. So this causes to formation of depletion zone or space charge region. According to Figure2, $\mathrm{E}_{\mathrm{V}}$ and $\mathrm{E}_{\mathrm{C}}$ indicate valence band and conduction band of energy level diagram respectively, which shows under forward bias, the potential barrier between p-type and n-type reduces. Also $E_{F}$ and $W_{D}$ indicate Fermi level and depletion width respectively. By current injection and applying a voltage in the forward direction of the device, electrons and holes are pushed to the junction, so the electron-hole pairs appear and recombine together, this result in the formation of light. The electrolumines- 
electrons by diffusion from the n-type region into the p-type region and combine with the acceptors. So causes to formation of depletion zone or space charge region. According to Figure2, $E_{V}$ and $E_{C}$ indicate vale band and conduction band of energy level diagram respectively, which shows under forward bias, the poter

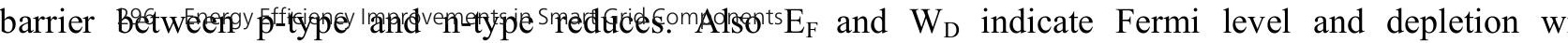
respectively. By current injection and applying a voltage in the forward direction of the device, electrons holes are pushed to the junction, so the electron-hole pairs appear and recombine together, this result in

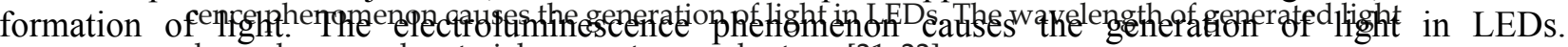

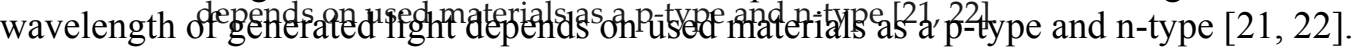

a

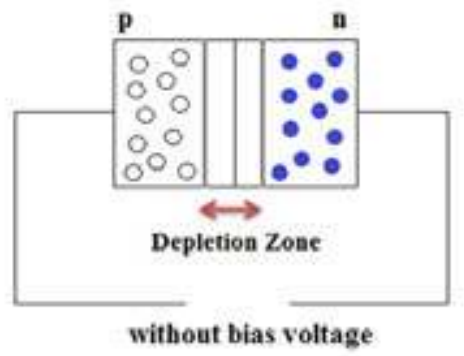

b

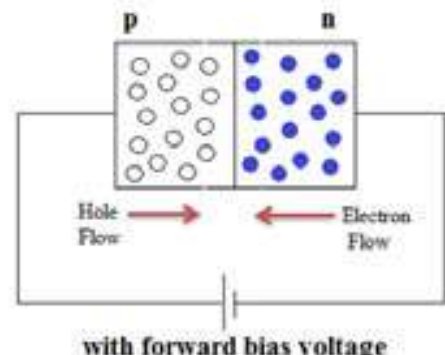

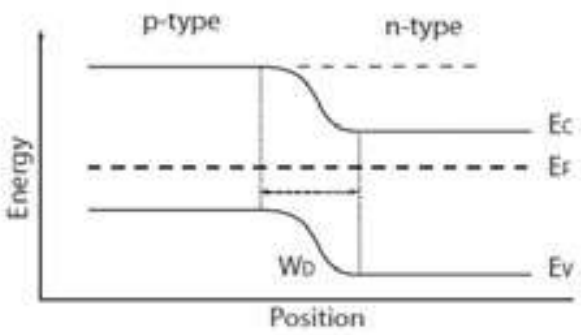

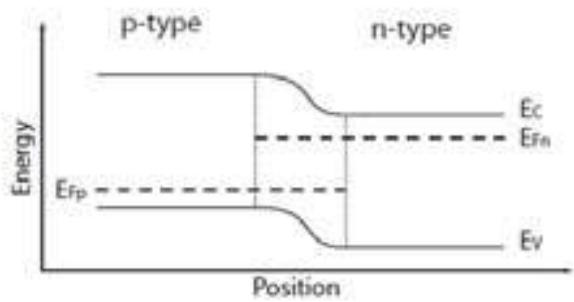

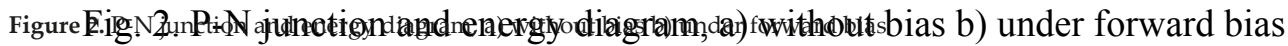

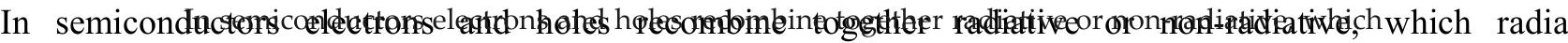

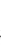

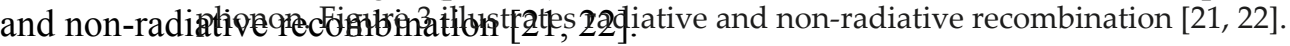
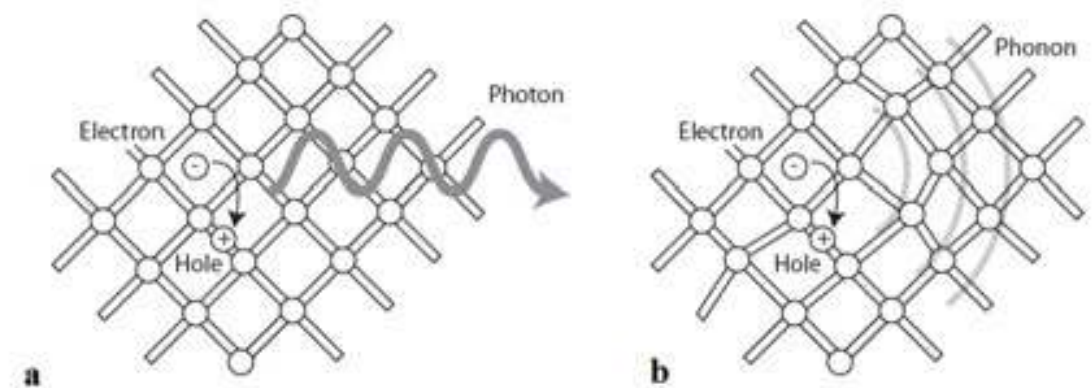

Fig. 3. a) Radiative recombination b) non-radiative recombination

Figure 3. a) Radiative recombination b) non-radiative recombination

Because of the presence of electron and hole in recombination process, this process is called bimolec recombination. The recombination rate, $\mathrm{R}$ is given by: 
Because of the presence of electron and hole in recombination process, this process is called bimolecular recombination. The recombination rate, $\mathrm{R}$ is given by:

$$
R=-\frac{d n}{d t}=-\frac{d p}{d t}=B n p
$$

where $\mathrm{B}$ is bimolecular recombination coefficient and $\mathrm{R}$ is proportional to the density of electron-hole pairs. In light emitting diodes non-radiative recombination is unwanted, because this process creates phonon, so the phonons causes to increase heat and heating of the material has harmful effects on emitted light and lifetime of device [21, 22]. QD-LEDs are another structure of LEDs that have multilayers and QD layer is an active layer. This LEDs consist of hole transport layer (HTL), electron transport layer (ETL) and QD layer as an emissive layer. In this type of LEDs, Indium tin oxide (ITO) (as an anode) and $\mathrm{Al}$ (as a cathode) use as usual electrodes. The HTL is contacted by ITO and ETL is contacted by Al, which acts as an injector [16]. In this device by applying forward bias and current injection, the holes and electrons are injected from anode and cathode respectively, and they are travel through of HTL and ETL to the QD layer, then they recombine together in QD layer [3, 16]. Figure 4 indicates QD-LEDs structure, energy level diagram, and physical performance of QD-LEDs. Forster resonant energy transfer (FRET) is excitation mechanism of QDs in close proximity to small organic molecules and inorganic layers. In FRET mechanism, in the charge transport layers formed electron-hole pairs transfer energy non-radiative to the QD layer by dipole-dipole coupling. Direct charge injection is the efficient method of injection carriers [3].

For explain the performance of QD-LEDs, these devices consist of five parts which listed as follow [16]:

a. Carrier injection from the electrodes

By assuming of position ( 0 and $\mathrm{w}$ ), and ohmic contacts at anode and cathode, the carrier concentrations inside the transport layers are equal to the equilibrium carrier concentrations that are given in Eq. 2.

$$
p(0) n(0)=n_{i}^{2}(0) \quad p(W) n(W)=n_{i}^{2}(W)
$$

At the electrodes, there is no space charge, so we have the following condition (Eq. 3).

$$
\begin{aligned}
& N_{d}(0)-N_{a}(0)+p(0)-n(0)=0 \\
& N_{d}(W)-N_{a}(W)+p(W)-n(W)=0
\end{aligned}
$$

where $\mathrm{n}$ and $\mathrm{p}$ are electron and hole concentrations respectively, $\mathrm{n}_{\mathrm{i}}$ is intrinsic carrier concentration, and $\mathrm{N}_{\mathrm{d}}$ is the donor concentration and $\mathrm{N}_{\mathrm{a}}$ is the acceptor concentration. At the electrodes, the electrostatic potential is given by the following equations (Eqs. 4 and 5). 
respectively, and they are travel through of HTL and ETL to the QD layer, then they recombine together in QD layer [3, 16]. Fig. 4 indicates QD-LEDs structure, energy level diagram, and physical performance of QD-LEDs. Forster resonant energy transfer (FRET) is excitation mechanism of QDs in close proximity to small organic

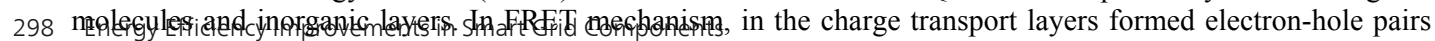
transfer energy non-radiative to the QD layer by dipole-dipole coupling. Direct charge injection is the efficient method of injection carriers [3].
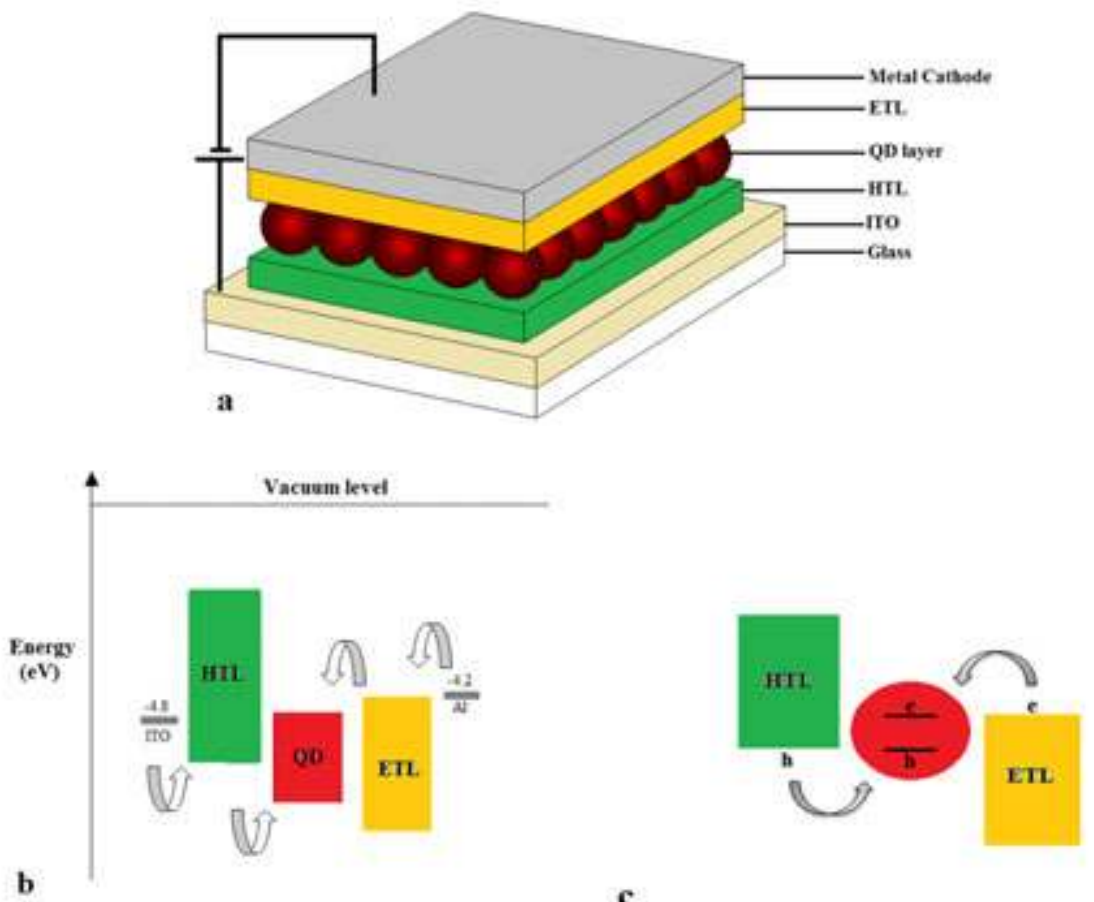

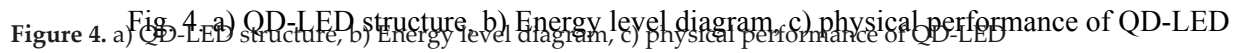

For explain the performance of QD-LEDs, these devices consist of five parts which listed as follow [16]:

a) Carrier injection from the electrodø $\left\{(0)=V-\frac{K T}{q} \ln \left[\frac{p(0)}{n_{i}(0)}\right]\right.$

$$
\psi(W)=\frac{K T}{q} \ln \left[\frac{n(W)}{n_{i}(0)}\right]-\left(\frac{\Delta E_{c}+\Delta E_{v}}{2}\right)
$$

According to Anderson's rule, the last term in equation, accounts for the discontinuities in the conduction band and valence band.

b. Transport in the ETL and HTL

The equations used to describe the transport in the HTL and ETL are similar to the bulk drift and diffusion equations, which is given as follows (Eqs. 6-10).

$$
\frac{d p}{d t}=-\frac{1}{q} \frac{d J_{p}}{d x}+G-U
$$




$$
\begin{gathered}
\frac{d n}{d t}=\frac{1}{q} \frac{d J_{n}}{d x}+G-U \\
\frac{d^{2} \psi}{d x^{2}}=-\frac{q}{\varepsilon}\left(N_{d}-N_{a}+p-n\right) \\
J_{p}=-q D_{p} \frac{d p}{d x}-q p \mu_{p} \frac{d \psi}{d x} \\
J_{n}=q D_{n} \frac{d n}{d x}-q n \mu_{n} \frac{d \psi}{d x}
\end{gathered}
$$

$\mathrm{G}$ indicates generation parameter which is assumed to be negligible and $\mathrm{U}$ is recombination rate and given by Shockley-Read-Hall (SRH) recombination, which will be described in (e) section.

c. Carrier injection from transport layers into the QD layers

Assuming that the QD layer adjacent to the HTL, the nearest QDs are considered to be traps for holes. In equilibrium and using detailed balance, the rate of emission is equal to the rate of captured holes by these QDs, so, we have (Eq. 11):

$$
e_{0} p_{0}(0)=c_{0} p_{0}\left(0^{-}\right)\left[N_{T}-p_{0}(0)\right]
$$

$\mathrm{p}(0)$ and $\mathrm{p}\left(0^{-}\right)$are hole density in the QD layer and HTL, which adjacent together, respectively. $\mathrm{N}_{\mathrm{T}}$ is the density of trap states in the QD layer (each QD can only accommodate one hole). $\mathrm{e}$ and $\mathrm{c}$ are emission rate coefficient and the capture rate coefficient for the trap states, respectively. Also subscript (0) is used for equilibrium condition. The ratio of emission and capture coefficients is given by Eq. 12 as follows.

$$
\frac{c_{0}}{e_{0}}=\frac{p_{0}(0)}{\left[N_{T}-p_{0}(0)\right] p_{0}\left(0^{-}\right)}
$$

The difference of capture and emission can be describes the rate of change of carrier concentration under non-equilibrium conditions, thus, we have (Eq. 13):

$$
\frac{d p}{d t}=c p\left(0^{-}\right)\left[N_{T}-p(0)\right]-e p(0)
$$


The capture and emission coefficients can be taken to be the same as for equilibrium, if device is close to equilibrium. Also if the carrier concentration is non-degenerate, the following equation manage rate of carrier change (Eq. 14) as:

$$
\frac{d p}{d t}=\frac{1}{\tau_{p 1}}\left[\frac{p\left(0^{-}\right)}{p_{0}\left(0^{-}\right)} p_{0}(0)-p(0)\right]
$$

where $\tau_{\mathrm{pl}}$ is capture/emission time constant and equals to $\frac{1}{\mathrm{e}}$, also $\tau_{\mathrm{pl}}$ depends on the used material. At the interface of ETL and QD layer, which are adjacent together, equations for electrons are entirely similar to holes.

d. Transport among the quantum dot layers

The QDs can be assumed semiconductor particles with a surrounded insulating layer. The transport from one QD to another occurs by direct tunneling process. In this case the QDs act as potential wells and the insulating layer acts as tunnel barriers. Using a one-dimensional WKB approximation, the tunneling probability from QD layer $1 \rightarrow 2$, is given by Eq. 15:

$$
T_{12}=e^{-2 \kappa\left(2 d_{i n s}\right)}
$$

where $\kappa$ is the inverse characteristic length for tunneling and $d_{\text {ins }}$ is the thickness of the insulating layer around the QDs. The electron is "oscillating" in the well with "frequency" $\vartheta=\frac{\vartheta_{\text {th }}}{2 \mathrm{~d}} \cdot \vartheta_{\text {th }}$ is the thermal velocity of electron, $\mathrm{d}$ is the diameter of the QD, and it has probability of $\mathrm{T}_{12}$ to making a transition to the neighboring QD layer. The total density of electrons per second tunneling from $1 \rightarrow 2$ assuming an unoccupied layer 2 are:

$$
N_{1 \rightarrow 2}=n_{1} \vartheta T_{12}
$$

where $n_{1}$ is the number of electrons in layer 1 . Because of the same size of the particles and temperature in layer 1 and 2, so the total number of electrons per second tunneling from 2 $\rightarrow 1$, assuming an unoccupied layer 1, is given by Eq. 17 as:

$$
N_{2 \rightarrow 1}=n_{2} \vartheta T_{21}
$$

Therefore the flow of electrons from layer 1 to layer 2 is given by Eq. 18 as:

$$
N_{1 \rightarrow 2}-N_{2 \rightarrow 1}=\vartheta\left(T_{12} n_{1}-T_{21} n_{2}\right)
$$


If $\mathrm{n}_{10}$ and $\mathrm{n}_{20}$ are equilibrium concentrations of electrons in layers 1 and 2 respectively, and $w_{12}=\vartheta \mathrm{T}_{12}$, so the net exchange is given by Eq. 19 as:

$$
N_{1 \rightarrow 2}-N_{2 \rightarrow 1}=W_{12}\left(n_{1}-\frac{n_{10}}{n_{20}} n_{2}\right)
$$

The equations of transporting of holes in QDs are similar to electrons equations.

e. Recombination in the QDs

In QD-LEDs like LEDs there are two types of recombination, radiative and non-radiative. If the recombination of electrons and holes are radiative, photons are emitted, and the wavelength of photon is independent to the charge transport layers and only depends on the QDs. According to doping of QDs, the radiative recombination can be either monomolecular or bimolecular. Monomolecular recombination occurs in doped quantum dots and the rate of recombination depends on minority carrier density. If the quantum dots are undoped, the recombination rate depends on both of the carriers, so this kind of recombination is bimolecular. Bimolecular recombination is given by Eq. 20 as:

$$
U_{r}=\gamma\left(n p-n_{i}^{2}\right)
$$

where $U_{r}$ is recombination rate, $\gamma$ is recombination rate coefficient, $n$ and $p$ are electron and hole concentrations respectively, $n_{i}$ is intrinsic carrier concentration. Also non-radiative recombination is given by Shockley-Read-Hall (SRH) recombination, as follow (Eq. 21).

$$
U_{n r}=\frac{p n-n_{i}^{2}}{\tau_{n}\left(p+n_{i}\right)+\tau_{p}\left(n+n_{i}\right)}
$$

where $\mathrm{U}_{\mathrm{nr}}$ is non-radiative recombination rate, $\mathrm{n}$ and $\mathrm{p}$ are electron and hole concentrations respectively, $n_{i}$ is intrinsic carrier concentration, also $\tau_{n}$ and $\tau_{p}$ are electron and hole recombination lifetimes.

\section{Experimental}

This section provides materials synthesis and fabrication methods of QD-LED devices that are realized by our research group. All of the fabricated devices consist of p-type and n-type materials as HTL and ETL, respectively. Synthesis of these materials will be explained in this section. 


\subsection{Synthesis of materials}

\subsubsection{P-type materials}

\subsubsection{Processing of $\mathrm{NiO}$}

$\mathrm{NiO}$ synthesized by electrochemical and sol-gel methods which is used in the structure of QDLED, also $\mathrm{ZnO}: \mathrm{Cu}$ is another material which is used as a HTL.

1. $\mathrm{NiO}$ film is fabricated using two electrode system at a deposition temperature of $50{ }^{\circ} \mathrm{C}$. An indium tin oxide ITO/glass and a Pt wire were used as the cathode and anode, respectively. The electrolyte was an aqueous solution containing $5 \mathrm{mmol}$ nickel nitrate and $5 \mathrm{mmol}$ hexamethylenetetramine (HMT). The voltage during deposition was-2.2 $\mathrm{V}$ and the deposition time was $30 \mathrm{~min}$ [23].

2. The $\mathrm{NiO}$ sol-gel precursor was prepared by dissolving $0.01 \mathrm{~mol}$ nickel nitrate in $20 \mathrm{ml}$ acetic acid and in a separate beaker $3 \mathrm{ml}$ tri-ethyleneamine dissolved in $30 \mathrm{ml}$ methanol and was added under stirring to the improve sol stability. The prepared gel were then placed immediately into a tube furnace and annealed under air at $600{ }^{\circ} \mathrm{C}$ for 2 hours, after that the gel changed to powder form. $0.01 \mathrm{~g}$ of obtained $\mathrm{NiO}$ dispersed in $2 \mathrm{ml}$ methanol and the dispersed solution were deposited on the ITO substrate at $100^{\circ} \mathrm{C}$.

\subsubsection{Processing of $\mathrm{ZnO}: \mathrm{Cu}$}

$\mathrm{ZnO}: \mathrm{Cu}$ synthesized by sol-gel method by the following manner. For preparing of this material, $3.1 \mathrm{~g}$ of zinc acetate and $0.18 \mathrm{~g}$ of $\mathrm{CuSO}_{4}$, were dissolved in $40 \mathrm{ml}$ distilled water, then $1.5 \mathrm{~g}$ of citric acid and $1.5 \mathrm{~g}$ of polyethylene glycol was added under stirring to the improve sol stability. The prepared gel were then placed immediately into a tube furnace and annealed under air at $600{ }^{\circ} \mathrm{C}$ for 15 hours, after that the gel changed to powder form.

\subsubsection{N-type materials}

1. The $\mathrm{ZnO}$ sol-gel precursor was prepared by dissolving $3.27 \mathrm{~g}$ zinc acetate in $40 \mathrm{ml}$ distilled water, then $1.5 \mathrm{~g}$ of citric acid and $1.5 \mathrm{~g}$ of polyethylene glycol was added under stirring to the improve sol stability. The prepared gel were then placed immediately into a tube furnace and annealed under air at $500^{\circ} \mathrm{C}$ for 15 hours, after that the gel changed to powder form.

2. ZnO:Ga was synthesized by solvothermal method using the following manner. In a typical experiment to synthesis $\mathrm{Zn}_{0.95} \mathrm{Ga}_{0.05} \mathrm{O}$ nanoparticles, $\mathrm{NaOH}(1 \mathrm{mmol})$, tri-octylphosphineoxide (TOPO, $5 \mathrm{mmol}), \mathrm{Zn}\left(\mathrm{CH}_{3} \mathrm{CO}_{2}\right)_{2}\left(\mathrm{H}_{2} \mathrm{O}\right)_{2}(0.95 \mathrm{mmol}), \mathrm{Ga}\left(\mathrm{NO}_{3}\right)_{3} \mathrm{H}_{2} \mathrm{O}(0.05 \mathrm{mmol})$, were mixed in $75 \mathrm{ml}$ 2-propanol and the mixture was transferred into autoclave. The autoclave was sealed and maintained at $180^{\circ} \mathrm{Cfor} 24$ hour, then allowed to cool to room temperature naturally. The obtained powder material is centrifuged by distilled water, ethanol and 2propanole for several times. 
3. $\mathrm{ZnO}: \mathrm{Nd}$ was synthesized by sol-gel method using the following manner. For preparing of $\mathrm{Zn}_{0.95} \mathrm{Nd}_{0.05} \mathrm{O}$, 3.1g of zinc acetate and $0.26 \mathrm{~g}$ of $\mathrm{NdCl}_{3}\left(6 \mathrm{H}_{2} \mathrm{O}\right)$, were dissolved in $40 \mathrm{ml}$ distilled water, then $1.5 \mathrm{~g}$ of citric acid and $1.5 \mathrm{~g}$ of polyethylene glycol was added under stirring to the improve sol stability. The prepared gel were then placed immediately into a tube furnace and annealed under air at $600^{\circ} \mathrm{C}$ for 15 hours, after that the gel changed to powder form.

\subsubsection{Quantum dots}

1. We utilized CdSe $\backslash \mathrm{ZnS}$ core $\backslash$ shell structures as quantum dots in the fabricated QD-LED. For synthesis of CdSe QDs, $\mathrm{Cd}\left(\mathrm{NO}_{3}\right)_{2}(3.24 \mathrm{mmol})$ and Oleic acid $(5 \mathrm{ml})$ were mixed together under vacuum conditions at $100^{\circ} \mathrm{C}$. Then Se $(1.62 \mathrm{mmol})$ dispersed in the 2propanol $(5 \mathrm{ml})$ and $7 \mathrm{mmol} \mathrm{NaBH}_{4}$ added in it and the obtained colorless solution were injected to the mixture of $\mathrm{Cd}\left(\mathrm{NO}_{3}\right)_{2}$ and oleic acid and heated at $100^{\circ} \mathrm{C}$ for $30 \mathrm{~min}$. The obtained material was centrifuged with n-hexane, ethanol and acetone for several times. The obtained CdSe capped with oleic acid was dried at $70^{\circ} \mathrm{C}$.Then $0.63 \mathrm{~g}$ of CdSe and $0.37 \mathrm{~g}$ of Zinc acetate solved in $10 \mathrm{ml} \mathrm{n}$-hexane and $10 \mathrm{ml}$ 2-propanol at $100^{\circ} \mathrm{C}$ under vacuum conditions. $0.02 \mathrm{~g} \mathrm{NaOH}$ and $0.05 \mathrm{~g}$ thioacetamide solved in $15 \mathrm{ml}$ 2-propanol and was added to the solution and the reaction was done for $30 \mathrm{~min}$ at $120^{\circ} \mathrm{C}$. The obtained material was centrifuged with n-hexane, ethanol, acetone and distilled water for several times.

2. CdS is another quantum dot, which has been used in the fabrication of QD-LED. For synthesis of CdS QDs, CdO (1mmol) and TOPO $(0.3 \mathrm{~g})$ and chloroform $(30 \mathrm{ml})$ were mixed together under vacuum conditions at $100^{\circ} \mathrm{C}$. Then $\mathrm{S}(2 \mathrm{mmol})$ dispersed in $2 \mathrm{ml}$ of Tri-tertbutylphosphine and this solution was injected to the mixture of $\mathrm{CdO}(1 \mathrm{mmol})$ and TOPO $(0.3 \mathrm{~g})$ and chloroform $(30 \mathrm{ml})$ at $100^{\circ} \mathrm{C}$ for $30 \mathrm{~min}$. The obtained material was centrifuged with n-hexane, ethanol and acetone for several times.

Surface of the synthesized CdS QDs for applying in QD-LEDs should be improved. For this purpose we got help from FRET mechanism and capping molecules for using as surface modification, selected based on enhancement of illumination of CdS using FRET mechanism. So we carried out surface modification of QDs by organic molecules, which listed as follow:

3. One of the materials for modification of QD surface is Thioacetamide (TAA). For preparing of CdS with TAA ligands, CdS (0.01g) capped with TOPO (synthesized QD) and TAA (0.01) and 2-propanol $(5 \mathrm{ml})$ were mixed together under stirring for 24 hours, after that the obtained material was centrifuged with 2-propanol, n-hexane, ethanol and distilled water for several times.

4. Ammonium hexafluorophosphate $\left(\mathrm{F}_{6} \mathrm{H}_{4} \mathrm{NP}\right)$ is another material which used as surface modification of QD. For preparing of CdS with this molecule, CdS $(0.01 \mathrm{~g})$ and $\mathrm{F}_{6} \mathrm{H}_{4} \mathrm{NP}$ $(0.01 \mathrm{~g})$ and 2-propanol $(5 \mathrm{ml})$ were mixed together under stirring for 24 hours, after that the obtained material was centrifuged with 2-propanol, n-hexane, ethanol and distilled water for several times.

5. Mercaptoacetic acid (MAA) is used as surface modification of CdS too. For preparing of $\mathrm{CdS}$ with Mercaptoacetic acid, $\mathrm{NaOH}(1 \mathrm{mmol})$ and Mercaptoacetic acid $(0.01 \mathrm{~g})$ and 2- 
propanol $(5 \mathrm{ml})$ were mixed together. Then the obtained solution was added on $0.01 \mathrm{~g}$ of the CdS and $5 \mathrm{ml}$ 2-propanol and the mixture stirred at room temperature for 24 hours; then the obtained material was centrifuged with 2-propanol, n-hexane, ethanol and distilled water for several times.

\subsection{Device fabrication}

This section provides seven kinds of QD-LEDs by using p-type, n-type and quantum dots materials which the synthesis of them were explained above. For fabrication of first, second and third QD-LEDs, ITO coated glasses with a sheet resistance of $20 \mathrm{ohm} / \mathrm{sq}$ was purchased from Aldrich. Also For fabrication of fourth, fifth, sixth and seventh, flexible ITO which was coated on PET was utilized. For fabrication of all of the devices, traditional physical method and solution processed method were utilized.

For fabrication of first QD-LED, NiO synthesized by sol-gel method as HTL layer was used. In this case CdSe/ZnS QD layer used as emissive layer and ZnO:Ga as ETL. All of applied materials dispersed in 2-propanole and coated by spin coating method.

For fabrication of second QD-LED, electrochemically synthesized $\mathrm{NiO}$ was deposited on ITO and CdSe/ZnS QD layer and ZnO:Ga as ETL layer were deposited on it by spin coating method respectively.

For fabrication of third QD-LED, ZnO:Cu as HTL layer was deposited on ITO and CdSe/ZnS QD layer was fabricated by spin coating method and ZnO:Nd as ETL layer was deposited on it by spin coating method respectively.

For fabrication of fourth, fifth, sixth and seventh QD-LED, sol-gel $\mathrm{NiO}$ as HTL, QD layer, $\mathrm{ZnO}: \mathrm{Nd}$ ETL were all deposited by spin coating method. The QD layer in fourth QD-LED is CdS capped with TAA molecule and in fifth QD-LED the QD layer consist of CdS capped with $\mathrm{F}_{6} \mathrm{H}_{4} \mathrm{NP}$ molecule, also the QD layer in sixth QD-LED is CdS capped with MAA, in seventh QD-LED the QD layer consists of CdS-TOPO.

In all seven devices, the $\mathrm{Al}$ cathode was deposited by the electron beam evaporation technique.

\subsubsection{Spectroscopic studies and structural characterization}

In this chapter all the measurements and characterizes carried out by the following devices:

Photoluminescence (PL) measurements were carried out by a Perkin-Elmer LS45 luminescence spectrophotometer. UV-Vis absorption spectra were recorded using a PG instrument T70 UV/ $\mathrm{V}$ is spectrophotometer. Surface morphology and distribution of the particles were studied via a TESCAN model MIR3 scanning electron microscope (SEM), and by a Dual-scope C26 scanning probe and microscope DME atomic force microscope (AFM) operating in tapping mode. The crystal structure of nanoparticles were characterized by X-ray diffraction (XRD) on a Siemens D500 using Cu-k $\alpha$ radiation $\left(\lambda=1.541 \mathrm{~A}^{\circ}\right)$. X-Ray photoelectron spectroscopy (XPS) was carried out by Surface Science Instruments (SSX-101 M-Probe ESCA). 


\section{Results}

\subsection{Increasing efficiency}

Because of the importance of light quality and lifetime of QD-LEDs, nowadays increasing efficiency of QD-LEDs is very considerable; therefore our group (OIC) by introducing new structures of QD-LEDs and using solution process method for fabrication of QD-LEDs has several works for improving of efficiency of this type of LEDs. In this way, crystal engineering and effects of FRET in QD-LED were investigated, which both of them improve quality of QDLEDs [24].

\subsubsection{Crystal engineering}

This section is based on trap level engineering in inorganic materials to achieve improved QD based LEDs. For this purpose, we investigate three types of QD-LEDs which in two types of them $\mathrm{NiO}$ applied as transparent, conductive hole transport layer (HTL) and CdSe/ZnS QDs as luminescent layer and $\mathrm{ZnO}: \mathrm{Ga}$ as electron transport layer (ETL). The arrangement of the layers that form the QD-LED is illustrated in Figure 5. As Figure 5a shows, there is an energy barrier for hole injection from ITO to the $\mathrm{p}-\mathrm{NiO}$ layer and from $\mathrm{p}-\mathrm{NiO}$ layer to the QD layer. Such a barrier is not present for electron injection and transportation. This causes a carrier density imbalance in QDs that prevents efficient recombination of electron-hole pairs. This phenomenon causes self-quenching in QD-LEDs. One way to solve this problem is synthesis of $\mathrm{NiO}$ nanoparticles with a wider band gap to decreasing energy barrier between $\mathrm{p}-\mathrm{NiO}$ and QDs. To improve hole injection from ITO to the $\mathrm{p}-\mathrm{NiO}$ layer, trap levels in the band gap of $\mathrm{NiO}$ are created that results in a much lower barrier for hole injection. As mentioned previously, engineering these trap energy levels is possible with considering different methods for nano structure synthesis. For this purpose, we investigate two types of $\mathrm{NiO}$ synthesized via sol-gel and electrochemical methods.

The purity and crystallinity of the $\mathrm{NiO}$ nanoparticles synthesized by sol-gel and electrochemical methods were examined using powder X-ray diffraction (XRD). XRD pattern of nanoparticles synthesized by sol-gel method is shown in Figure 6 which is similar to the pattern for material synthesized by electrochemical method. This figure shows narrow diffraction peaks which indicate high crystallinity of the synthesized materials and the peaks appeared at $2 \theta=37.39^{\circ}, 43.38^{\circ}, 62.94^{\circ}$ are related to $(111),(200),(220)$ crystal planes of the synthesized $\mathrm{NiO}$ which crystallizes in cubic system. Metal-Oxides like NiO usually contain a large number of defects and these defects convert the $\mathrm{NiO}$ to conductive material. In equilibrium defect chemistry, $\mathrm{NiO}$ usually has an oxygen excess accommodated by nickel vacancies $[25,26]$. To preserve the overall electrical neutrality in the crystal, some $\mathrm{Ni}^{2+}$ ions must be converted to $\mathrm{Ni}^{3+}$ ions, which are responsible for conduction in $\mathrm{NiO}$. Lattice defects are not well-defined in $\mathrm{NiO}$ films synthesized by different methods. On the other hand, the vacancy model is little discussed with regard to $\mathrm{NiO}$ films prepared by different methods. Due to its uncertain mechanism most papers use nickel vacancy and/or interstitial oxygen to explain the electrical properties of the NiO films [26]. 


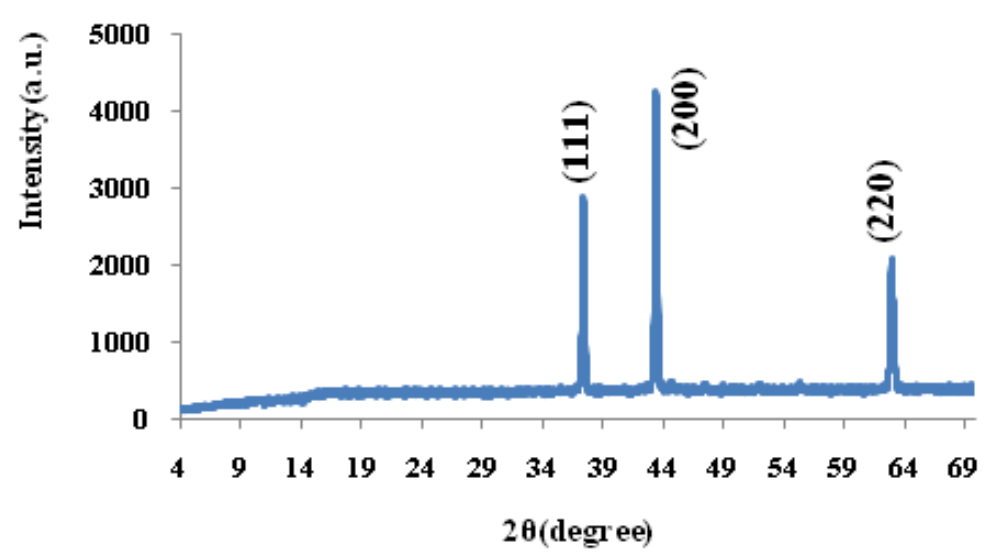

Figure 6. XRD pattern of $\mathrm{NiO}$ nanoparticles

the crystalline defects in the $\mathrm{NiO}$ structures and is related to intrinsic defects and $\mathrm{Ni}^{2+}$ vacancies play a key role for it.

As figures show, intensity of the emission bands appeared in visible range for the material synthesized by sol-gel method is higher than electrochemical method that indicates density of trap levels in the material synthesized by so-gel method is high. Absorption spectra for synthesized materials show broad bands in the range of 190-350 nm for the material synthesized by electrochemical method which is narrower in the material synthesized by sol-gel method. This result indicates high uniformity of the particles synthesized by sol-gel method. On the other hand appearing the band at $335 \mathrm{~nm}$ for the material synthesized by sol-gel method shows a blue shift rather than electrochemically synthesized material which this peak appears at $350 \mathrm{~nm}$. This result shows creation of wide band gap material with sol-gel method.

Figure 9 shows the scanning electron microscope (SEM) images of the synthesized $\mathrm{NiO}$ using sol-gel and electrochemical methods which indicates uniform and smaller size of particles for $\mathrm{NiO}$ synthesized by sol-gel method. AFM images (Figure 10) confirm smooth surface and uniform size of $\mathrm{NiO}$ synthesized by sol-gel method.

Intrinsic defects are created during crystallization process of $\mathrm{ZnO}$ and by this way n-type $\mathrm{ZnO}$ can obtain [27-30]. In previously reported devices $\mathrm{ZnO}$ with intrinsic n-type defects has been used. We tried to fabricate such devices with extrinsic defects that are created by $\mathrm{Ga}$ or $\mathrm{Nd}$ doping in the structure of $\mathrm{ZnO}$. The reason for choosing these atoms as dopant atoms will describe in DFT calculation section. Since $\mathrm{ZnO}$ is crystalizes as n-type semiconductor, the number of p-type $\mathrm{ZnO}$ is limited and synthesis of this type of $\mathrm{ZnO}$ is not usual. Here we describe the devices based on $\mathrm{Cu}$ doped $\mathrm{ZnO}$ as the HTL beside devices based on p-type $\mathrm{NiO}$ which is applied as HTL layer in these structures. 


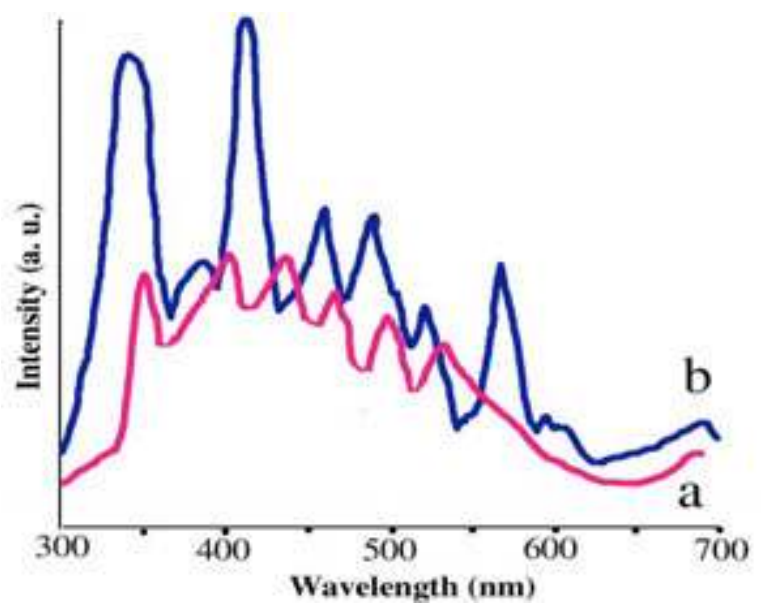

Figure 7. PL spectra of the synthesized NiO by: a) electrochemical and b) sol-gel methods
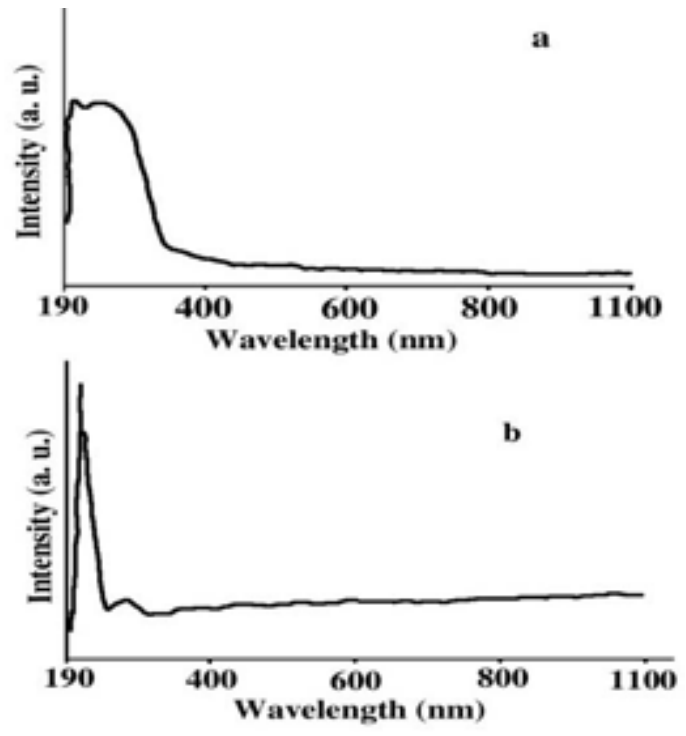

Figure 8. UV-Vis spectra of $\mathrm{NiO}$ synthesized by a) electrochemical and b) sol-gel methods.

Figure 5a shows, electron injection between $\mathrm{Al}$ (as electrode)- $\mathrm{ZnO}$ and $\mathrm{ZnO}-\mathrm{QD}$ depends on electronic structure of $\mathrm{ZnO}$. Although there is a small energy barrier in this case, increasing electronic levels in conduction band of $\mathrm{ZnO}$ can improve electron transport between layers. As calculations show, with doping of $\mathrm{Ga}^{3+}$ in the structure of $\mathrm{ZnO}$ electronic levels are created in the band structure of $\mathrm{ZnO}$ which these new levels appears inside the conduction band (Figure 11). 


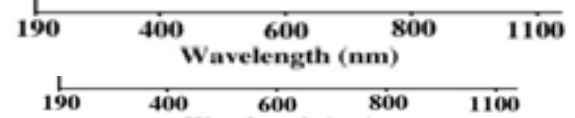

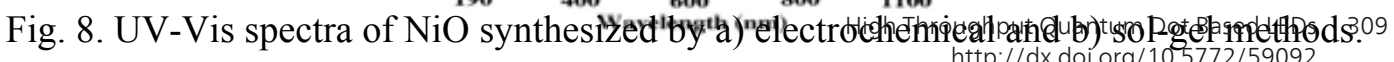

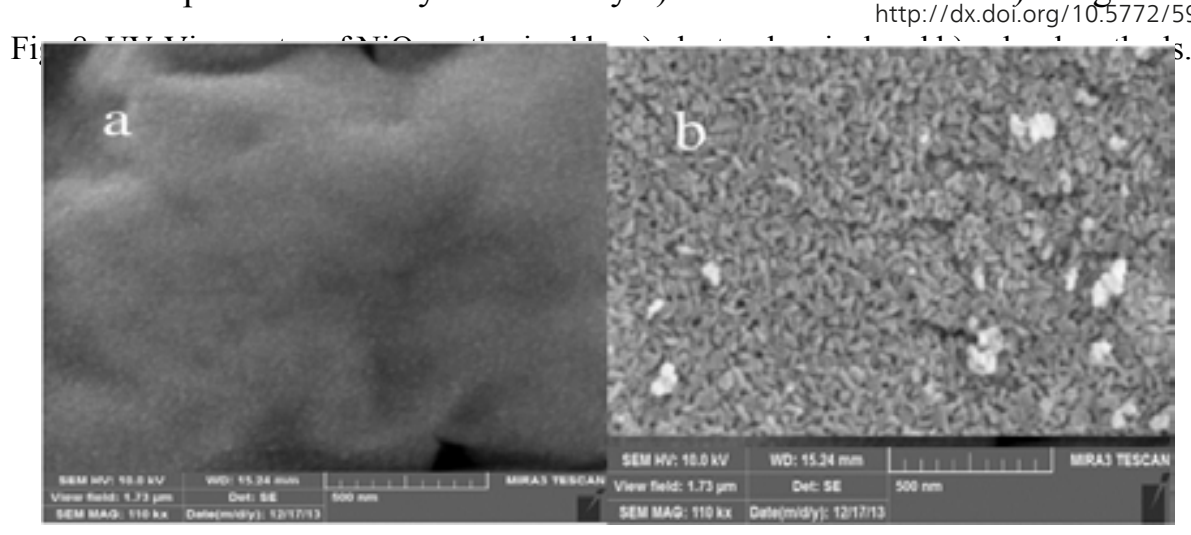

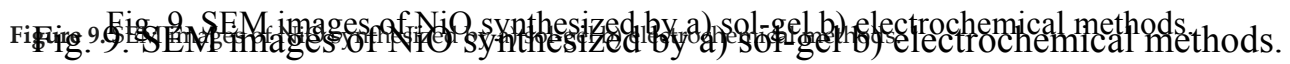

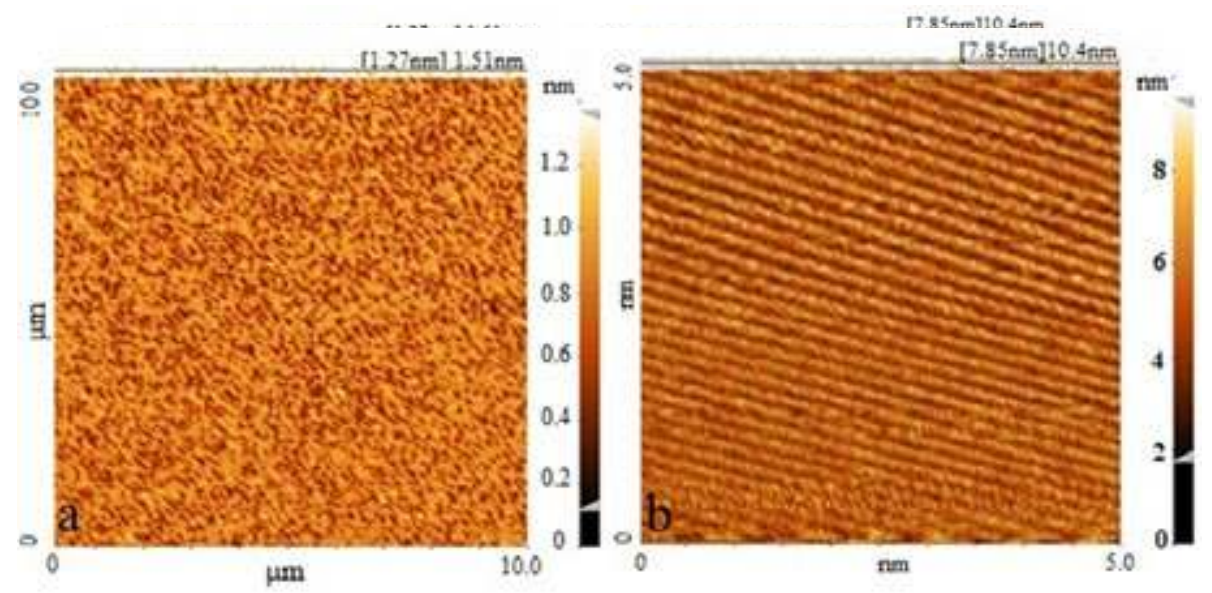

Fig. 9 shows the scanning electron microscope (SEM) images of the synthesized NiO using sol-gel and

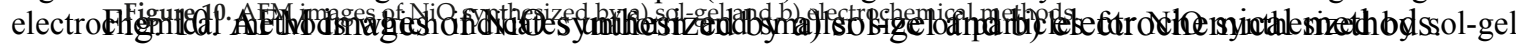
method. AFM images (Fig. 10) confirm smooth surface and uniform size of NiO synthesized by sol-gel method.

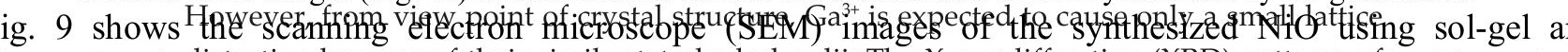

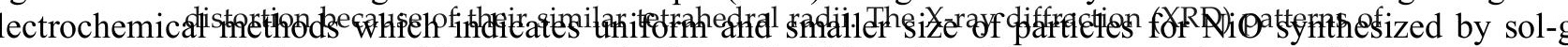

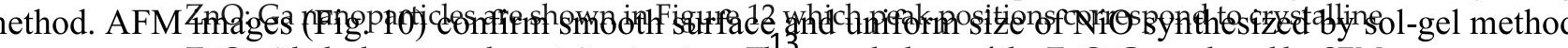
$\mathrm{ZnO}$ with the hexagonal wurtzite structure. The morphology of the $\mathrm{ZnO}$ : Ga analyzed by SEM and AFM images (Figure 13). These images confirm the small size of $\mathrm{ZnO}: \mathrm{Ga}$ nanoparticles which are about 50nm. The surface composition of films was determined using XPS technique. The XPS spectra of the ZnO: Ga films (Figure1Bl) show that the binding energy (BE) of each constituent element was positioned at $1117.72 \mathrm{eV}\left(\mathrm{Ga} 2 \mathrm{p}_{3 / 2}\right), 1022.23 \mathrm{eV}\left(\mathrm{Zn} 2 \mathrm{p}_{3 / 2}\right)$ and $530.9 \mathrm{eV}$ $\left(\mathrm{O}_{1 \mathrm{~s}}\right)$ as calibrated to $285.43 \mathrm{eV}\left(\mathrm{C}_{1 \mathrm{~s}}\right)$. The Ga-doped $\mathrm{ZnO}$ sample prepared using the $5 \mathrm{wt}$ \% $\mathrm{Ga}\left(\mathrm{NO}_{3}\right)_{3}$ reveals $4.75 \% \mathrm{Ga}$ on the surface of the films. The broadening of oxygen spectrum (Figure 13) is believed to be composed of two components located around $531 \mathrm{eV}$ and $532 \mathrm{eV}$ respectively. The low $\mathrm{BE}$ component is ascribed to covalently bonded oxygen in $\mathrm{ZnO}$ structure (lattice oxygen) while the high BE is attributed to the adsorbed oxygen. The higher binding 

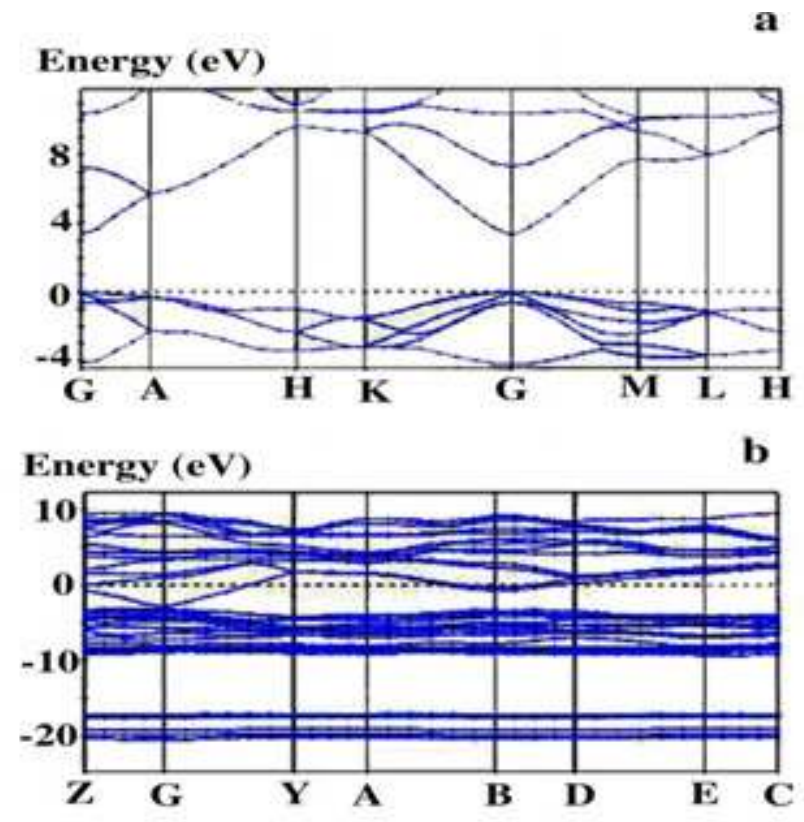

Figure 11. a) Band structure a) bare and b) Ga doped $\mathrm{ZnO}$

energy at $532 \mathrm{eV}$ is usually attributed to chemisorbed or dissociated oxygen or $\mathrm{OH}$ species on the surface of the $\mathrm{ZnO}$ thin film, such as adsorbed $\mathrm{H}_{2} \mathrm{O}$ or adsorbed $\mathrm{O}_{2}$ [31].

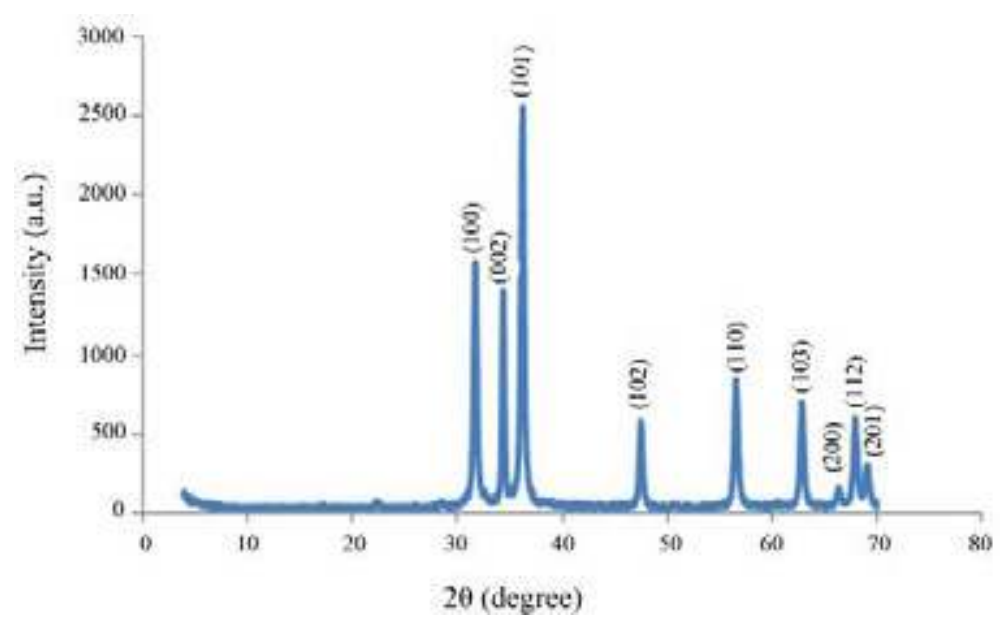

Figure 12. XRD pattern of $\mathrm{ZnO}: \mathrm{Ga}$ nanoparticles 


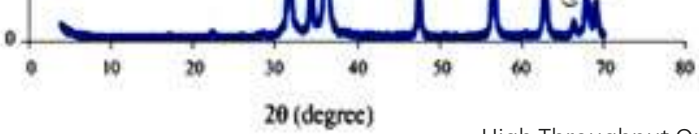

Fig. 12. XRD pattern of ZnO:Ga nanoparticles
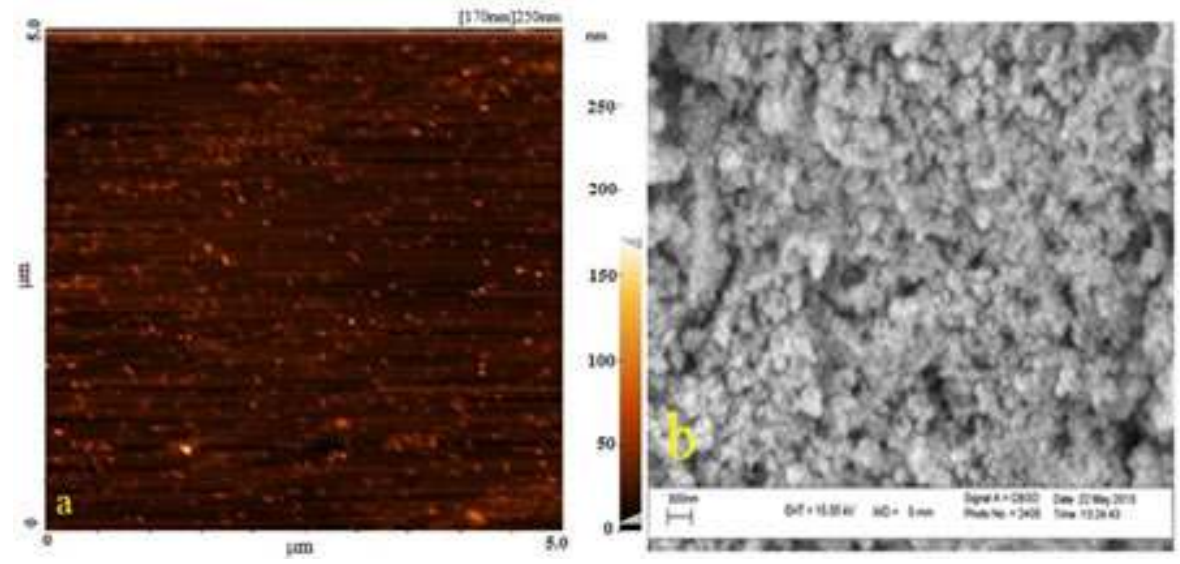

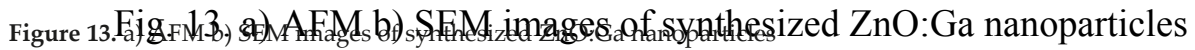

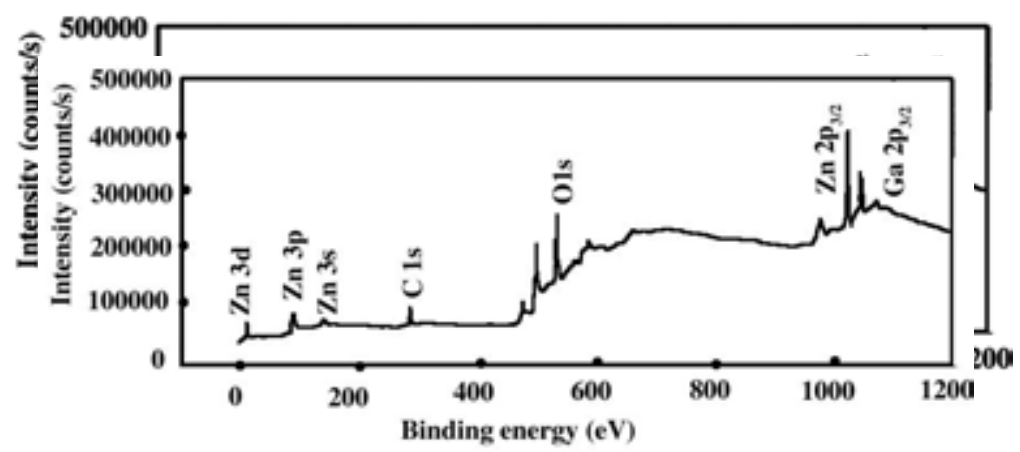

Fig. 14. XPS spectra of the $\mathrm{ZnO}$ : Ga films

Figure 14. XPS spectra of the ZnO: Ga films

L study of this thin $\mathrm{ZnO}$ film indicates high degree of surface and structural trap levels characterized by visibl PL study of this thin ZnO film indicates high degree of surface and structural trap levels characterized by visible-region fluorescence (Figure 15).

$\mathrm{Nd}$ doped $\mathrm{ZnO}$ has been synthesized previously by Zheng and coworkers [32]. DFT calculations show, doping of $\mathrm{Nd}$ in the structure of $\mathrm{ZnO}$ increases electronic levels in conduction band of $\mathrm{ZnO}$ (Figure 16) and these levels are more than levels in $\mathrm{Ga}$ doped $\mathrm{ZnO}$ structure which is expected that this structure will improve the efficiency of LED more than previous structure.

Figure 17 shows the $\mathrm{X}$-ray diffraction (XRD) patterns of $\mathrm{ZnO}$ : Nd nanoparticles. Peak positions correspond to crystalline $\mathrm{ZnO}$ with the heجggonal wurtzite structure. For $\mathrm{Zn}_{0.95} \mathrm{Nd}_{0.05} \mathrm{O}$, diffraction peaks become broader and weaker compared to undoped $\mathrm{ZnO}$ which shows decreasing of crystallinity of the structure by increasing in doping concentration. The morphology of the ZnO: Nd analyzed by AFM images (Figure 18). The grain sizes of the nanoparticles were $50-70 \mathrm{~nm}$. 


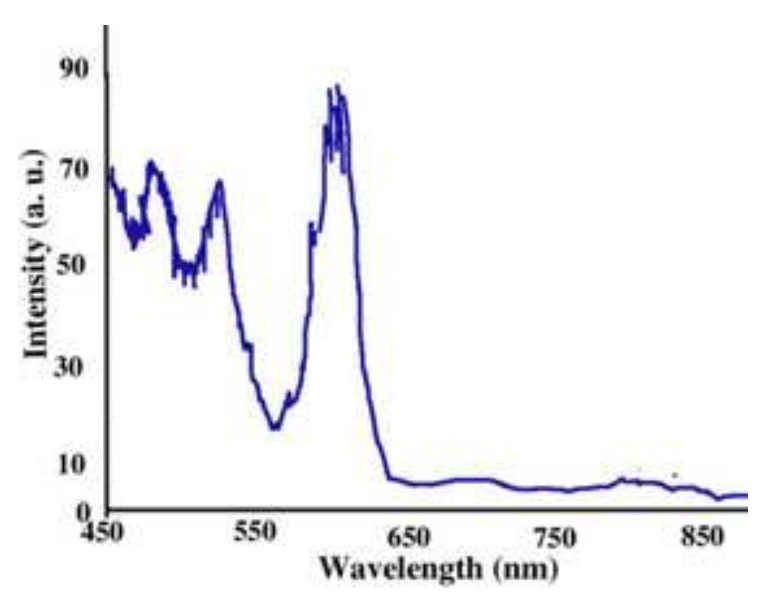

Figure 15. PL spectra of the synthesized $\mathrm{ZnO}$ :Ga nanoparticles

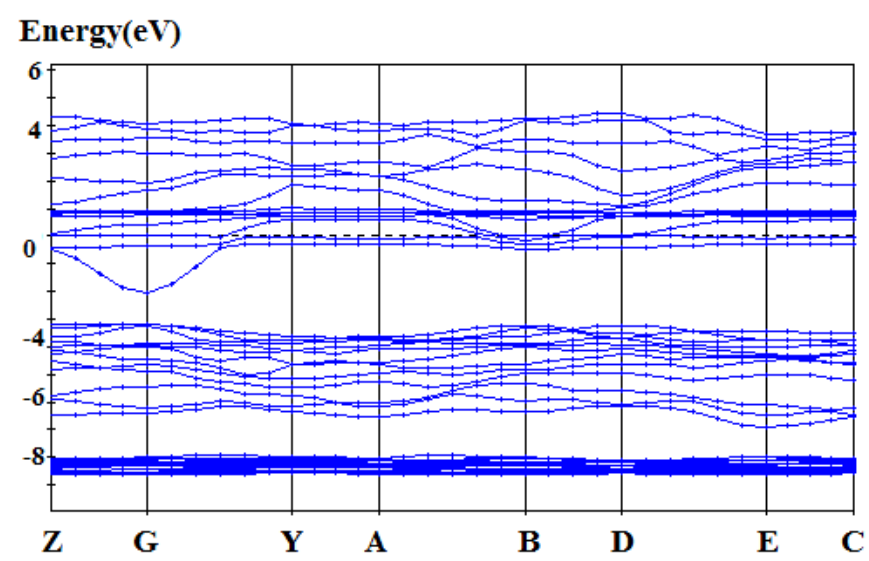

Figure 16. Band structure of $\mathrm{Nd}$ doped $\mathrm{ZnO}$

For making improvement in HTL layer, a structure based on Cu doped $\mathrm{ZnO}$ (instead of p-type $\mathrm{NiO}$ ) is fabricated. Doping of $\mathrm{Cu}^{2+}$ has effect on photoluminescence (PL) and structure of $\mathrm{ZnO}$ [33]. Calculation results indicate that with doping of $\mathrm{Cu}^{2+}$ in the structure of $\mathrm{ZnO}$ electronic levels are created in the band structure of $\mathrm{ZnO}$ which these new levels appears inside the valence band. Since electronic configuration in $\mathrm{Cu}^{2+}$ is $\mathrm{d}^{9}$, so, there is a level in created levels that is half full and can act as hole transfer. Figure 19 indicates the band structure of $\mathrm{ZnO}: \mathrm{Cu}$.

To get the information about the morphology and size of the $\mathrm{ZnO}: \mathrm{Cu}$ nanoparticles, AFM studies had been carried out. It is observed from the AFM images, shape of the nanoparticles 


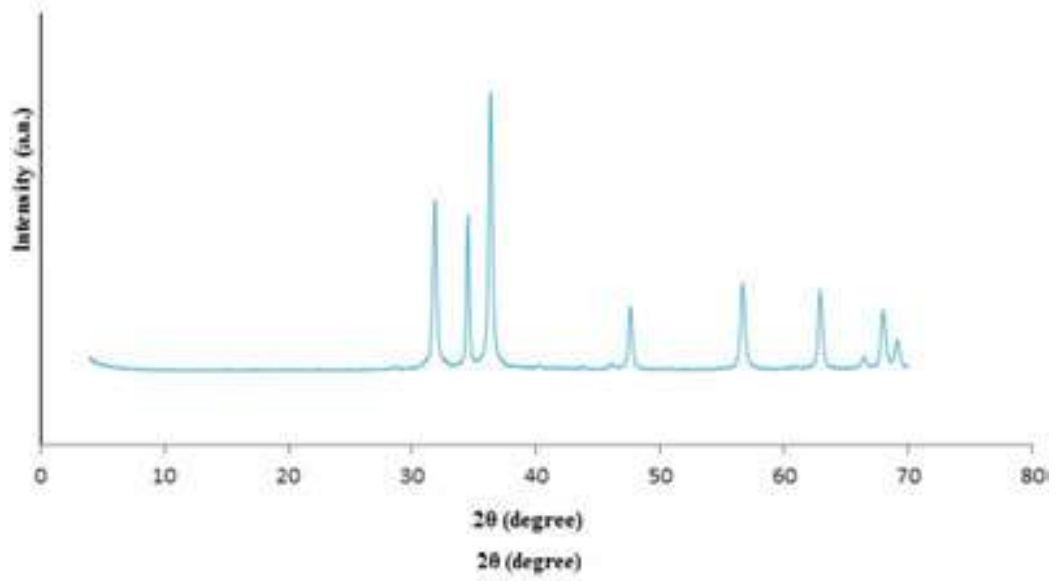

Fig. 17. XRD pattern of $\mathrm{ZnO}$ : Nd nanoparticles

Figure 17. XRD pattern of $\mathrm{ZnO}: \mathrm{Nd}$ nanoparticles
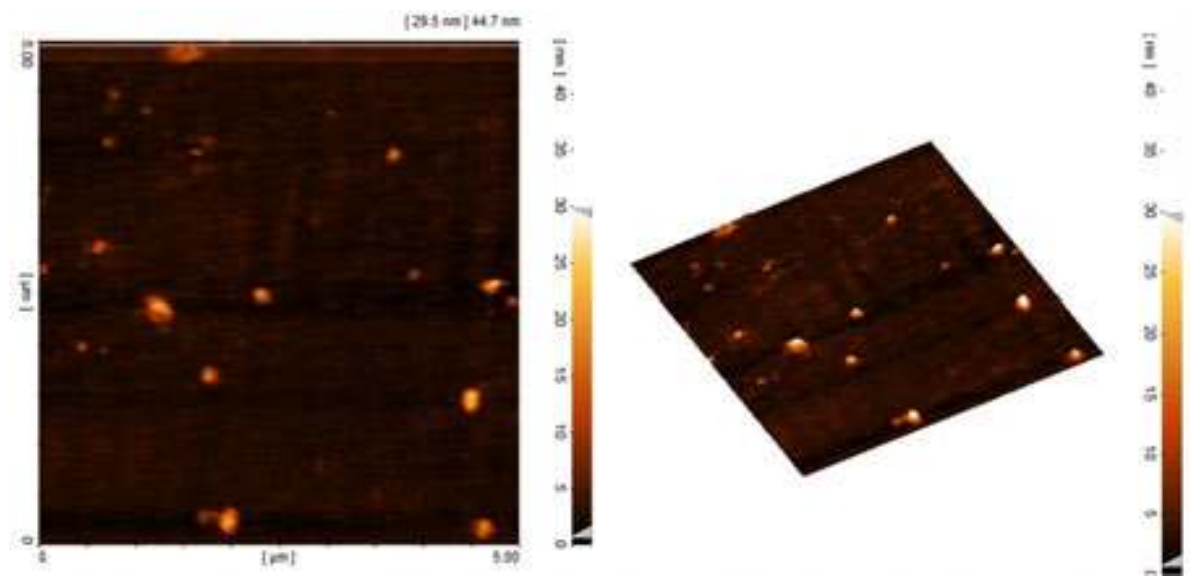

Figure 18. AFM images of $\mathrm{ZnO}: \mathrm{Nd}$

Fig. 18. AFM images of $\mathrm{ZnO}: \mathrm{Nd}$

For making improvement in HTL layer, a structure based on $\mathrm{Cu}$ doped $\mathrm{ZnO}$ (instead of p-type $\mathrm{NiO}$ ) is

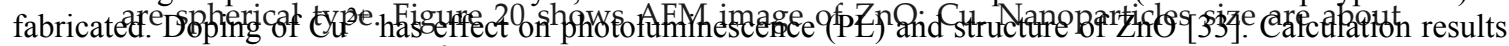
indicate $\mathrm{th}$. $\mathrm{ZnO}$ which these new levels appears inside the valence band. Since electronic configuration in $\mathrm{Cu}^{2+}$ is $\mathrm{d}^{9}$, so,

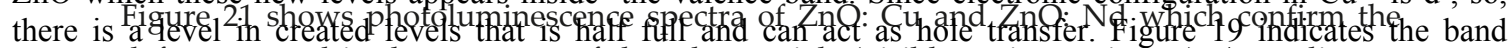
structuredefeztocreated in the structure of doped materials (visible region emission). According to

Figure 21 in both of the doped (Cu and $\mathrm{Nd}) \mathrm{ZnO}$ there is a peak in the $\mathrm{UV}$ region which shows band edge of $\mathrm{ZnO}$.

The colloidal QDs employed in our synthesized structures contain a core/ shell; CdSe/ZnS. Figure 22 shows PL spectra of CdSe/ZnS nanoparticles that demonstrate high emission peak at $600 \mathrm{~nm}$. The absence of other peaks in the spectrum and relatively narrow emission band at $600 \mathrm{~nm}$ show uniformity of particles and good passivation of the surface of CdSe using ZnS 


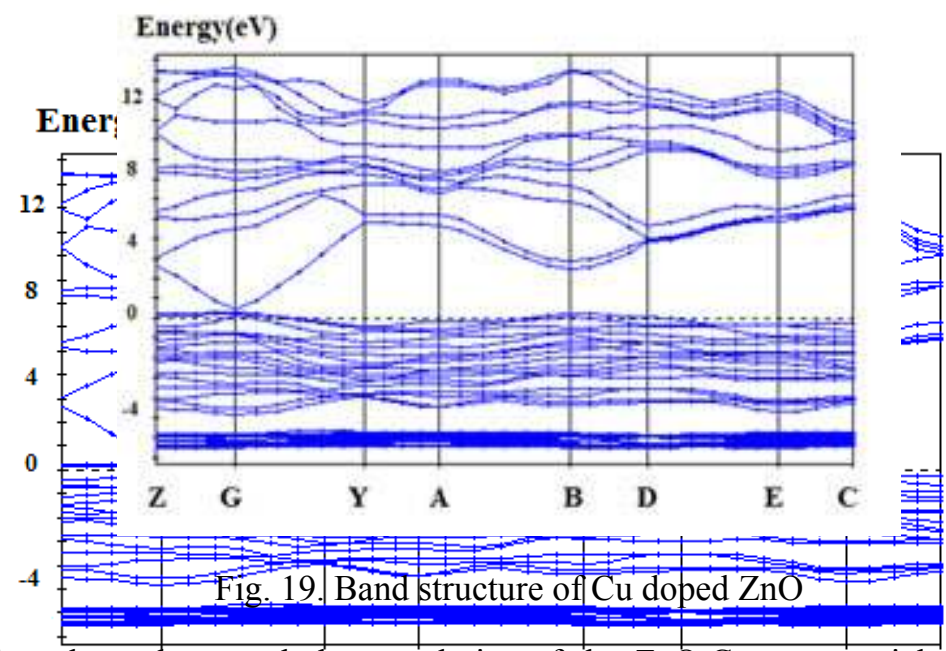

To get the information about the morphology and size of the $\mathrm{ZnO}: \mathrm{Cu}$ nanoparticles, AFM studies had been carried out. It is observed from the AFM images, shape of the nanoparticles are spherical type. Fig. 20 shows AFM image of $\mathrm{ZnO}$ : $\mathrm{Cu}$. Nanoparticles size are about 10-40nm.

Figure 19. Band structure of $\mathrm{Cu}$ doped $\mathrm{ZnO}$

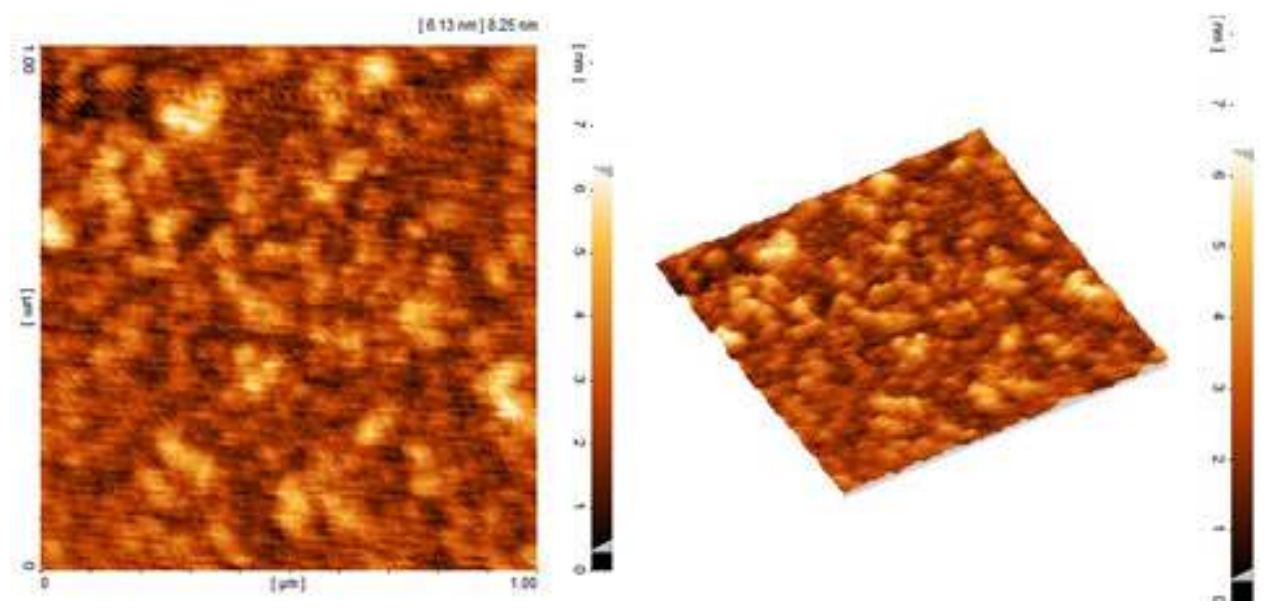

Figure 20. AFM images of $\mathrm{ZnO}: \mathrm{Cu}$

Fig. 20. AFM images of $\mathrm{ZnO}: \mathrm{Cu}$

Fig. 21 shows photoluminescence spectra of ZnQ. $\mathrm{Cu}$ and ZnO: Nd which confirm the defects created in the structure of dhell. The SEM. image of synthesized Case Zn core/shell material is ill istrated in Figure $23 \mathrm{Cu}$ and $\mathrm{Nd}$ ) $\mathrm{ZnO}$ there in a peak in the UV region which shows band edge of $\mathrm{ZnO}$.

The PL spectra of QD-LEDs are shown in Figure 24 and indicate that electron-hole recombination is occurring predominantly in the QD layer, as required for optimal device operation. $\mathrm{I}-\mathrm{V}$ behavior of the devices is illustrated in Figure 25 which confirms PL and EL emission results. Turn-on voltage for the devices based on $\mathrm{NiO}$ nano-materials is about $1 \mathrm{~V}$ which is higher than the device based on $\mathrm{ZnO}$ : $\mathrm{Cu}$. This turn-on voltage demonstrates that an ETL such 

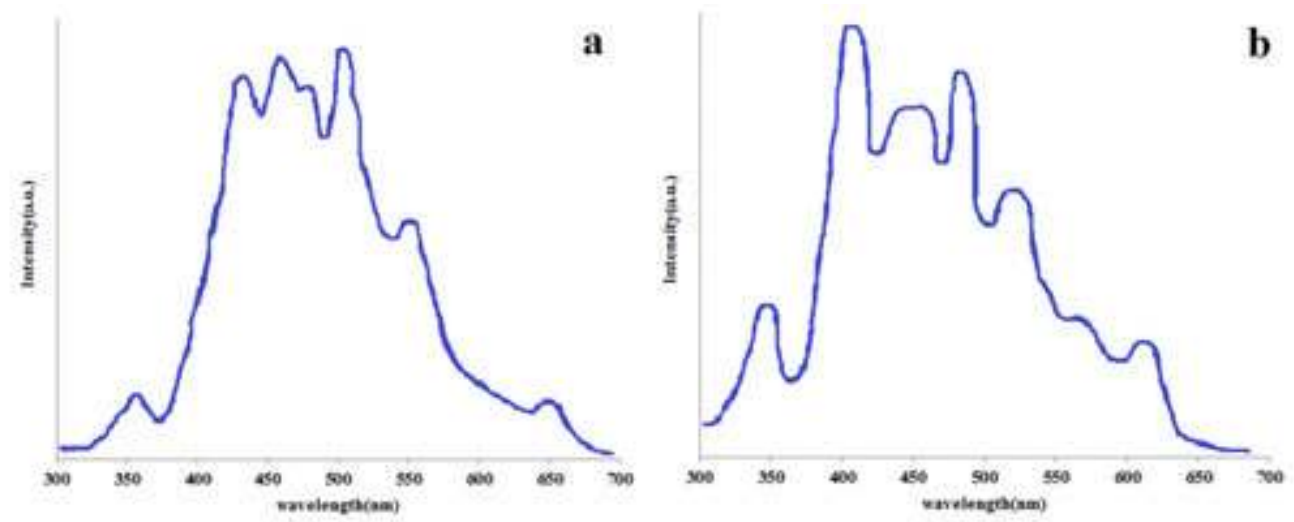

Figure 21. a) PL of $\mathrm{ZnO}: \mathrm{Cu}$ b) PL f5ign $(\mathrm{H} \mathrm{Hal}$ ) PL of $\mathrm{ZnO}: \mathrm{Cu}$ b) PL of $\mathrm{ZnO}: \mathrm{Nd}$

The colloidal QDs emploved in our svnthesized structures contain a core/ shell: CdSe/ZnS. Fig. 22 shows PL spectra of $\mathrm{CdSe} / \mathrm{ZnS}$ ne in the spectrum and $r$ passivation of the surfar is illustrated in Fig. $23 \mathrm{i}$

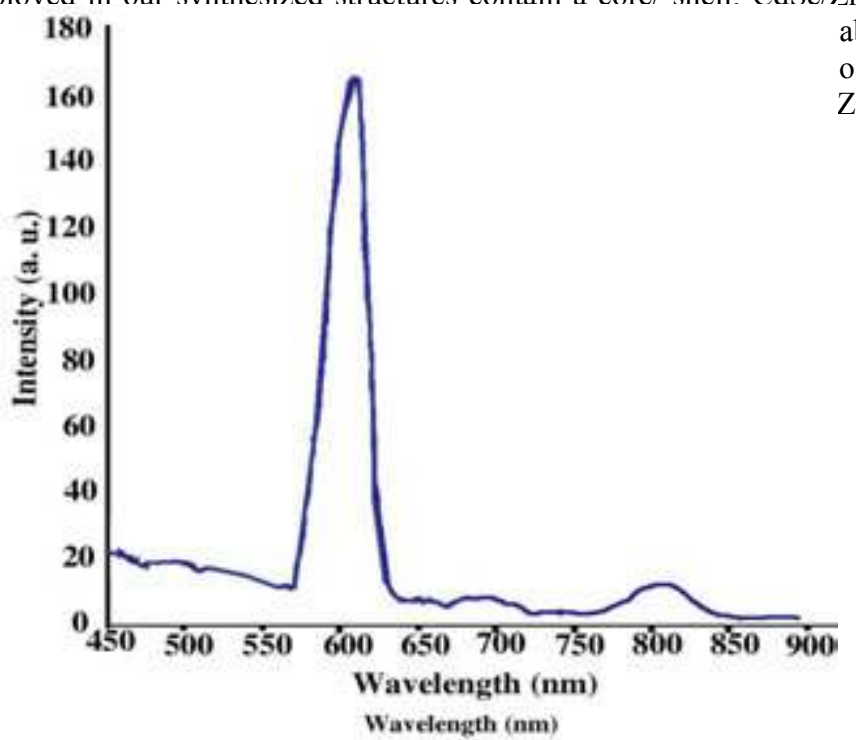
absence of other peaks of particles and good $\mathrm{ZnS}$ core/shell material

Figure 22. PL spectrum of the synthesized CdSe/ZnS

Fig. 22. PL spectrum of the synthesized CdSe/ZnS

as $\mathrm{ZnO}$ : $\mathrm{Ga}$ and $\mathrm{ZnO}: \mathrm{Nd}$ and $\mathrm{NiO} \mathrm{HTL}$ or $\mathrm{ZnO}$ : $\mathrm{Cu}$ facilitate effective electron and hole injection into the QD conduction and valence bands. According to Figure 25a and 25b measurements of the EL emission intensity yield a peak brightness of $500 \mathrm{cdm}^{-2}$ and $340 \mathrm{cdm}^{-2}$ at an applied operating bias of $5 \mathrm{~V}$ for fabricated LEDs based on materials synthesized by sol-gel and electrochemical methods respectively, which is considerable compared with other recently reported QD-LEDs. This brightness is 700 $\mathrm{cdm}^{-2}$ for the device based on $\mathrm{ZnO}: \mathrm{Nd}$ (as ETL layer) and $\mathrm{ZnO}: \mathrm{Cu}$ (as HTL layer) which the result confirms by I-V curve. As we can see in Figure 25 the current for it is higher than others. Also the current for the device based on $\mathrm{NiO}$ 
synthesized by sol-gel method is higher than $\mathrm{NiO}$ synthesized by electrochemical method. This shows that by engineering in the structure of QDLEDs their performance can be improved.

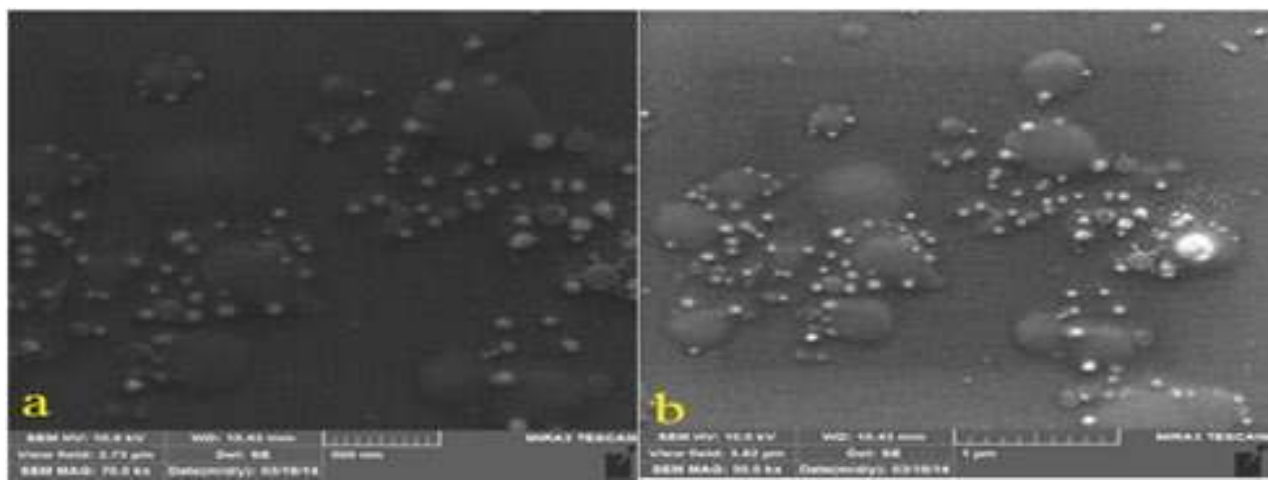

Figure 23. SEM imageFdigy 2 hesißeddimagessof synthesized CdSe/ZnS QDs

ne PL spectra of QD-LEDs are shown in Fig. 24 and indicate that electron-hole recombination is occurrir edominantly in the QD layer, as required for optimal device operation. I-V behavior of the devices ustrated in Fig. no-materials is at an ETL $\operatorname{such}_{3}$ so $^{\circ}$ jection into the $\epsilon^{3}$ nission intensity bricated LEDs nsiderable com $\mathrm{ZnO}: \mathrm{Nd}$ (as E Fig. 25 the cur
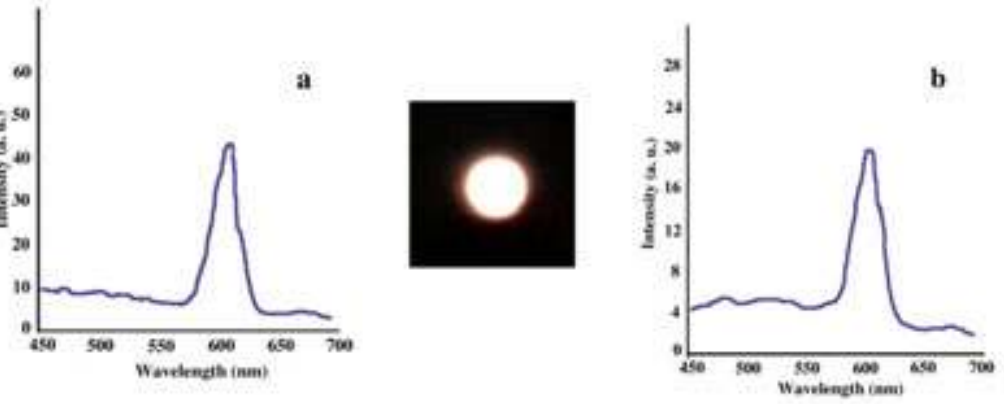
jased on $\mathrm{Ni}$ demonstrat

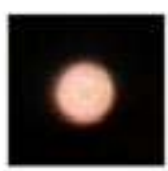
tron and ho ints of the $\mathrm{E}$ as of $5 \mathrm{~V} \mathrm{fo}$ rely, which device base ts we can se ynthesized $\mathrm{b}$ 1-gel method is higher than NiU synthesized by electrochemical method. I his shows that by engineering in th cucture of QDLEDs their perform

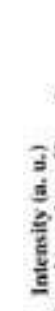

हn

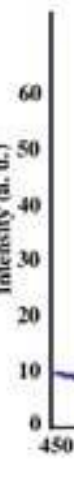

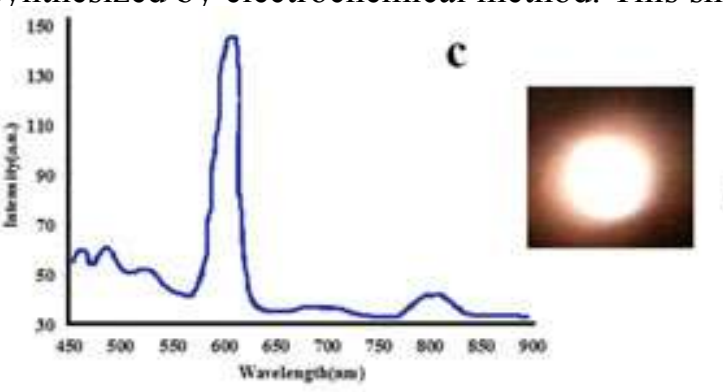

b

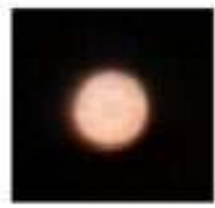

Fig. 24. $\mathrm{PL}$ spectra and observed light for fabricated QD-LẾps a) with $\mathrm{NiO}$ synthesized by sol-gel b) with $\mathrm{NiO}$

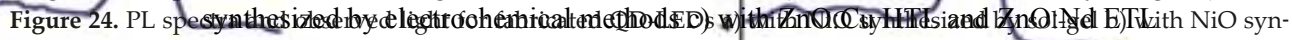
00 thesized by electrochemical methods c) with $\mathrm{ZnO}: \mathrm{Cu}$ HTL and $\mathrm{ZnO}$. Wavelength (nm)

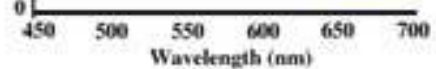



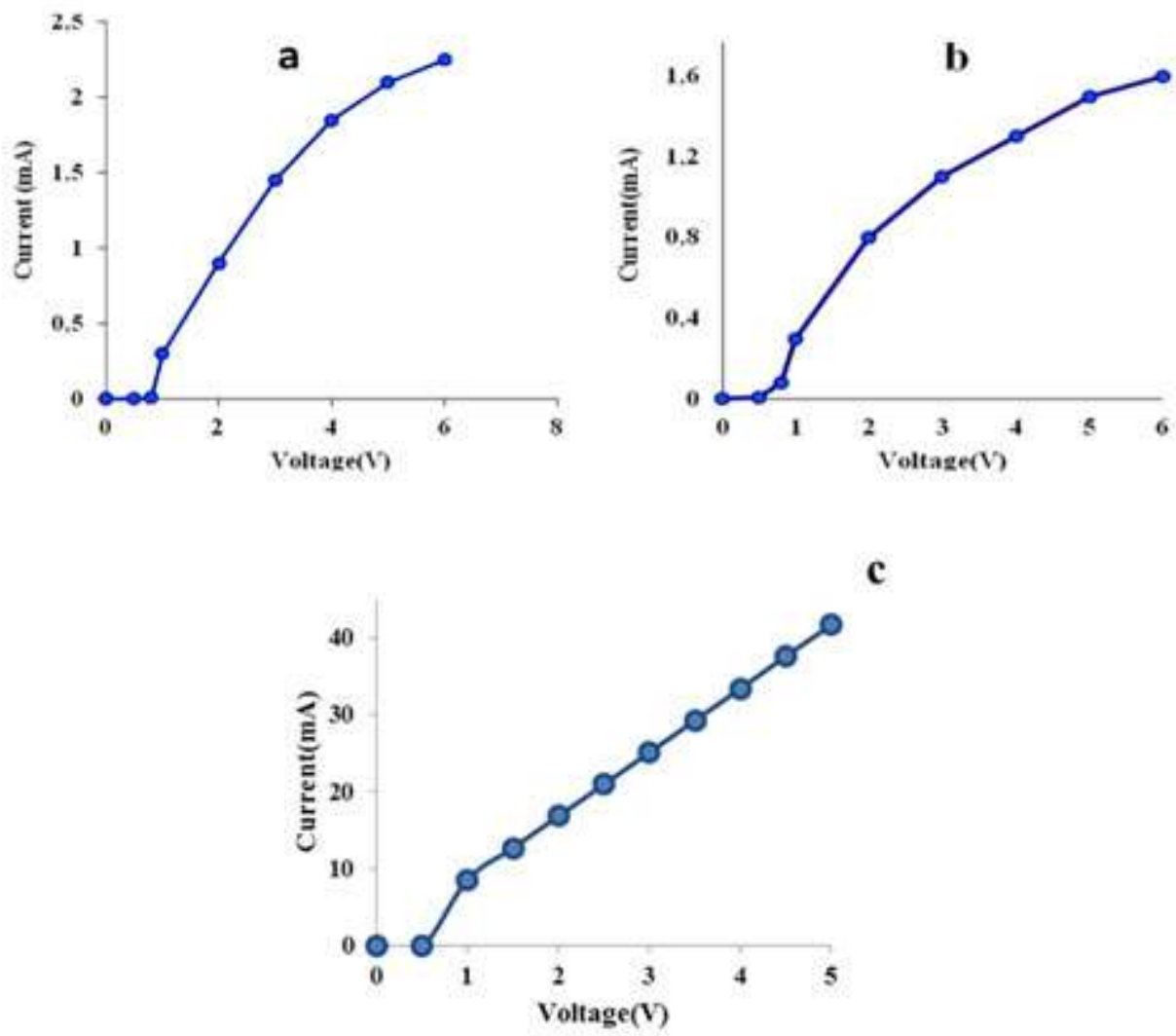

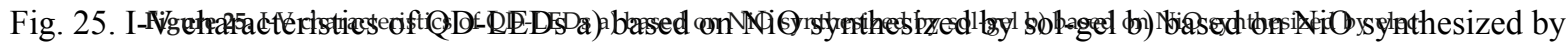
trochemicellectrod

\subsubsection{Effects of FRET in QD-LEDs}

\subsubsection{Effects of organic molecules as shell around QDs}

The emitted light and efficiency of QD-LEDs can be increased by Forster resonance energy

4.1.2) Effectsoff FRRTTin QDAIsEDy organic materials act as capping molecules for inorganic QDs.

FRET is an energy transfer between two molecules, which one of them is a donor and the

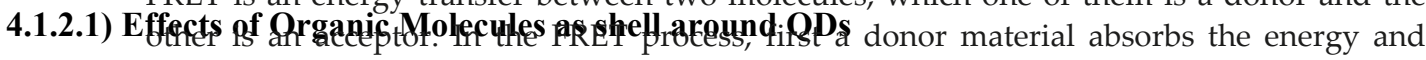

The emitted lightransfer absorbed engroy to a nearby acceptor and this process occurs through long-

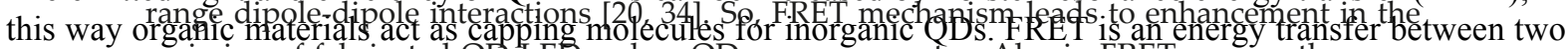

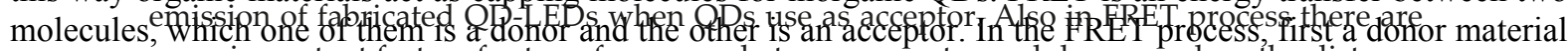

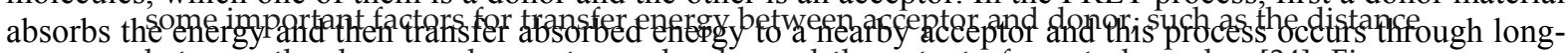

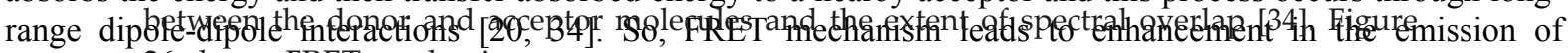

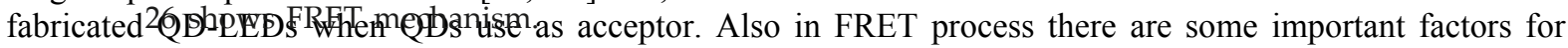
transfer energy between acceptor and donor, such as the distance between the donor and acceptor molecules and the extent of spectral overlap [34]. Figure 26 shows FRET mechanism.

$D+h \vartheta \rightarrow D^{*}$ 


$$
\begin{aligned}
& D+h \vartheta \rightarrow D^{*} \\
& D^{*}+A \rightarrow D+A^{*} \text { (D is Donor and A is Acceptor) } \\
& A^{*} \rightarrow A+h A^{\prime}
\end{aligned}
$$

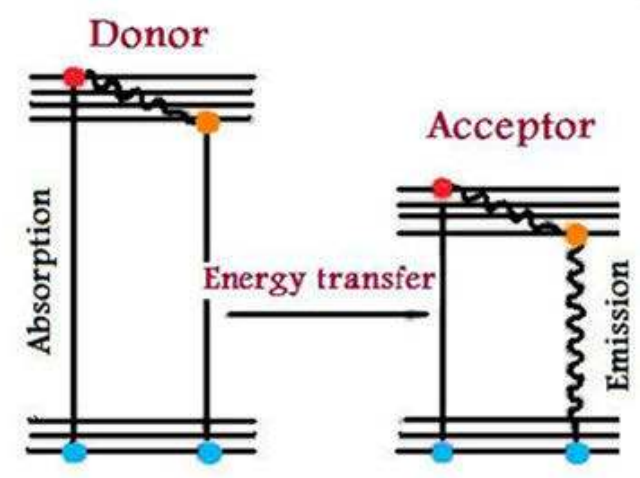

Figure 26. Energy diagram illustrating the FRET process

In the devices with luminescent species like inorganic semiconductors and organics molecules which in close proximity to the QDs, FRET is a mechanism that is unique to these devices. So, at first in the luminescent species, an exciton formed, and then the excitons energy is transferred to a QD non-radiative by dipole-dipole coupling [3]. QDs exhibit tune able emission by controlling their size and structure, therefor the spectral tune ability of QDs with quantum confinement effect allows to control FRET energy flow [35]. In our research group we utilized different capping organic molecules synthesized for surface passivation of synthesized QDs and these materials used as an active layer in the fabrication of QD-LEDs. The PL spectra of these materials indicate enhancement of emitted light via FRET mechanism. Figure 27 shows PL spectra of CdS QD and modified CdS QDs.

Figure 27 shows Intensity of emitted light for the CdS passivated by Ammonium hexaflurophosphate is higher than TAA and the PL intensity of CdS passivated by TAA is higher than CdS passivated by MAA. In this structure organic molecules are as donors and QDs are as acceptors. In FRET process the suitable distance for transmission of energy is about 1 to $10 \mathrm{~nm}$, so for generating of an energy gradient structure and occurring of FRET mechanism the donor organic molecules should be close to the acceptors (QDs). The trapped exciton can be transferred to the nearby QD and a fraction of the migrated excitons from trapped states can contribute to the acceptor luminescence by radiative recombination. So, this leads to enhancement in the emission. Figure 28 illustrates I-V behavior of the devices, fabricated by capped QDs; turn on voltage of three devices is about $1 \mathrm{~V}$, however, for the device based on CdS: TAA the turn on voltage is 1.5 volt, which is more than the other devices. This is while; the PL result of QD capped by TAA is higher than others. 

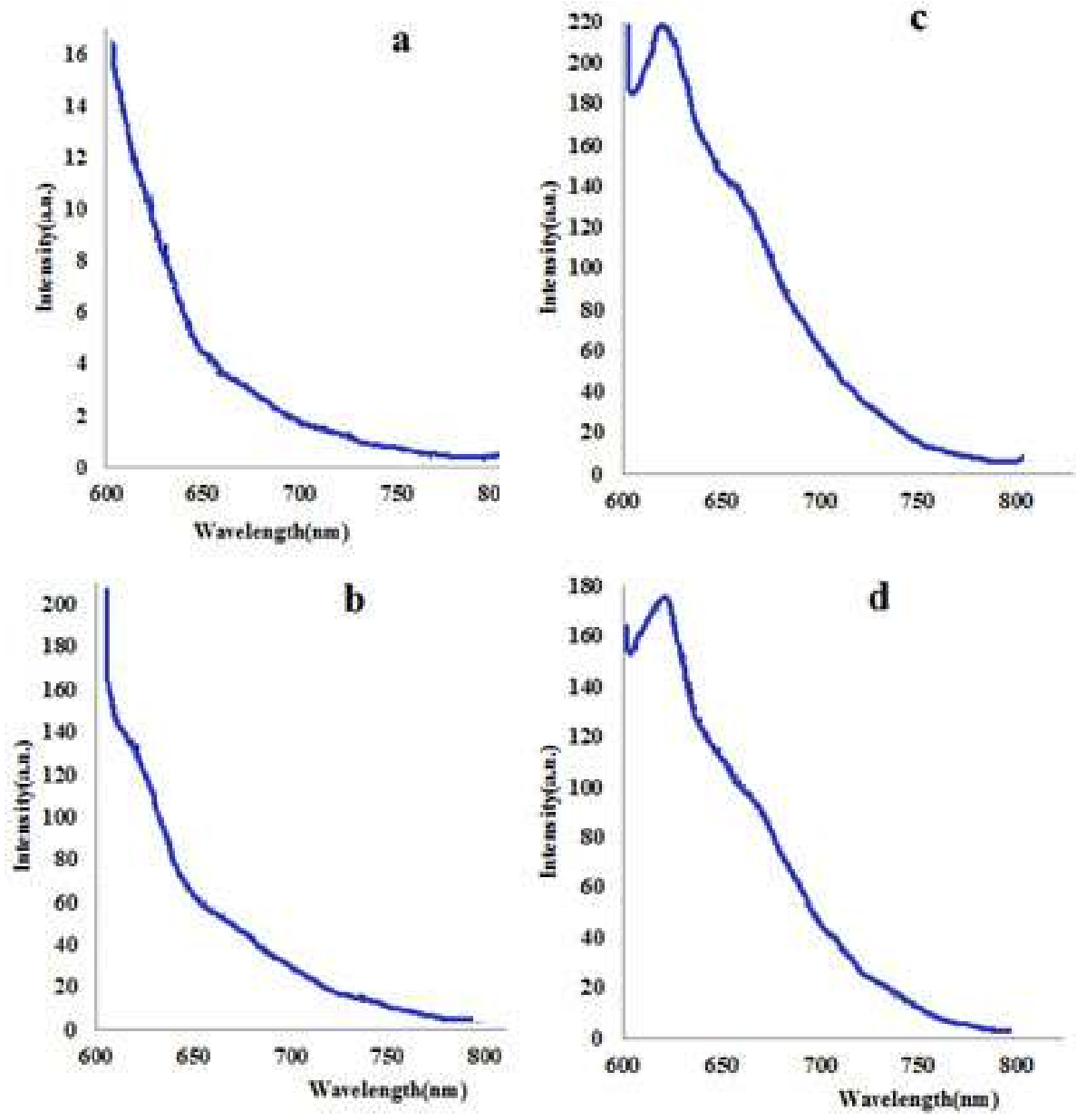

Figure 27. PL spectra of a)CdS b)CdS passivated by Ammonium hexafluorophosphate c) CdS passivated by TAA d) CdS passivated by MAA 

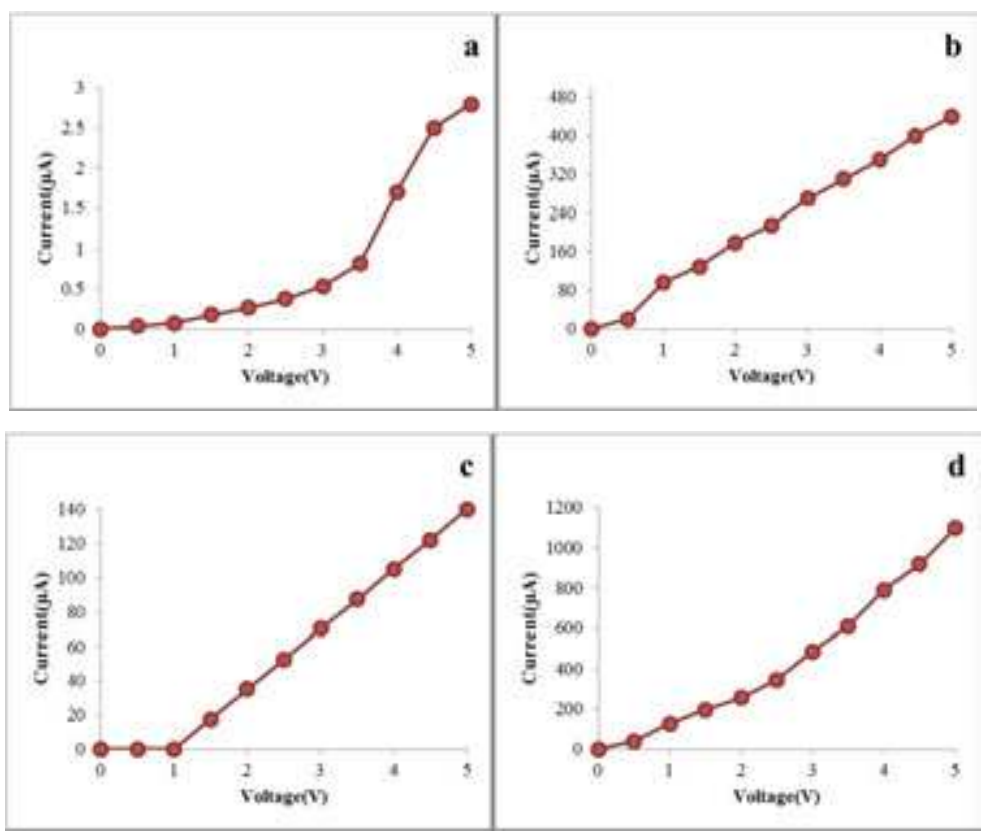

Fig. 28. I-V behavior of a) CdS b) CdS passivated by Ammonium hexafluorophosphate c) CdS passivated by

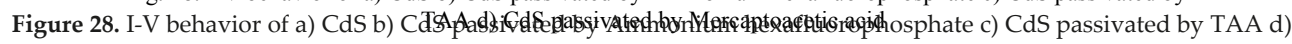
CdS passivated by Mercaptoacetic acid

(in

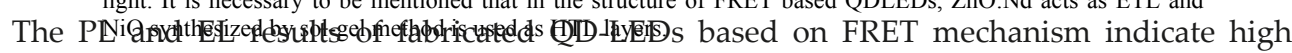
intensisycof minitited dighspectivesnecessary to be mentioned that in the structure of FRET based

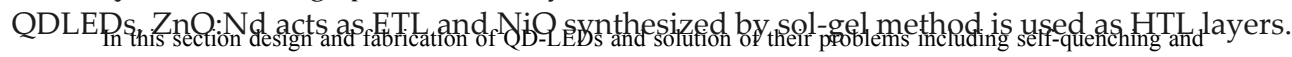
carrier injection have been described. All of described devices are fabricated by solution-processed methods. The base is finding the materials with suitable defect levels for achieving improved QD based LEDs. For this purpose, we investigated three types of QD-LEDs, which in both of them $\mathrm{NiO}$ applied as the transparent,

5. Conductive hole transport layer (HTL), and in the other one $\mathrm{ZnO}: \mathrm{Cu}$ is the $\mathrm{HTL}$. However, in one device, the
cons and persipectises in the other, the electrochemical synthesis route is used. Characterization of $\mathrm{NiO}$ synthesized by sol-gel and electrochemical methods showed that different trap levels are created in the crystalline structures of NiO. Also DFT calculations indicate doping of Gallium and Neodymium

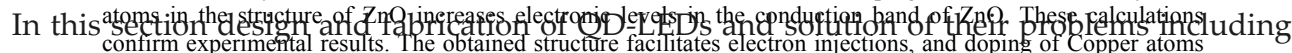

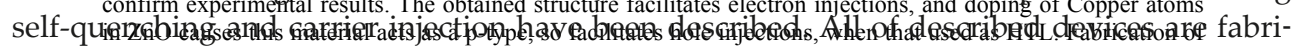
cated by solution-processed methods. The base is finding the materials with suitable defect levels for achieving improved QD based LEDs. For this purpose, we investigated three types of QD-LEDs, which in both of them $\mathrm{NiO}$ applied as the transparent, conductive hole transport layer (HTL), and in the other one $\mathrm{ZnO}: \mathrm{Cu}$ is the HTL. However, in one device, the synthesis route of $\mathrm{NiO}$ was sol-gel, whereas in the other, the electrochemical synthesis route is used. Characterization of $\mathrm{NiO}$ synthesized by sol-gel and electrochemical methods showed that different trap levels are created in the crystalline structures of $\mathrm{NiO}$. Also DFT calculations indicate doping of Gallium and Neodymium atoms in the structure of ZnO increases electronic levels in the conduction band of $\mathrm{ZnO}$. These calculations confirm experimental results. The obtained structure facilitates electron injections, and doping of Copper atoms in $\mathrm{ZnO}$ causes 
this material acts as a p-type, so facilitates hole injections, when that used as HTL. Fabrication of FRET based QD-LEDs could introduce suitable organic capping molecules for fabrication of high performance LEDs. In this way Ammonium hexafluorophosphate as capping molecule acts the best performance from view point of PL and EL results. Increasing in drive voltage and created heating in the light emitting diodes cause that the intensity of output light decrease and this is inefficient. In this work by introducing novel structures and improvement in the optical properties of materials used in devices, drive voltage and so, creating of heating in the fabricated devices is decreased so the light efficiency increases.

\section{Acronym list}

Light emitting devices; LEDs

high-intensity discharge; HID

Quantum dots; QDs

Hole transport layer; HTL

Electron transport layer; ETL

Indium tin oxide; ITO

Forster resonant energy transfer; FRET

Shockley-Read-Hall; SRH

Photoluminescence; PL

Scanning electron microscope; SEM

Atomic force microscope; AFM

X-ray diffraction; XRD

X-Ray photoelectron spectroscopy; XPS

Binding energy; BE

Polyethylene terephthalate; PET

\section{Author details}

P. Amini, M. Dolatyari*, G. Rostami and A. Rostami

*Address all correspondence to: m.dolatyari@tabrizu.ac.ir

OIC Research Group, School of Engineering-Emerging Technologies, University of Tabriz, Tabriz, Iran 


\section{References}

[1] Mills E., "The $\$ 230$-billion global lighting energy bill”. In Proceedings of the $5^{\text {th }}$ International Conference on Energy-Efficient Lighting, Nice., France., May 2002.

[2] Yang Y., Pei Q., and Heeger A.J., “Efficient blue light-emitting diodes from a soluble poly (para-phenylene): internal field emission measurement of the energy gap in semiconducting polymers". Synthetic Metals. 1996; 78: 263-267.

[3] Shirasaki Y., Supran G. J., Bawendi M. G., and Bulović V., "Emergence of colloidal quantum-dot light-emitting technologies". Nat. Photonics. 2013; 7: 13-23.

[4] Round H. J., “A note on carborundum”. Electr. World. 1907; 47: 309.

[5] Hall R. N., Fenner G.E., Kingsley J., Soltys T. J., and Carlson R. O., “Coherent light emission from GaAs junctions". Phys. Rev. Lett., 1962; 9: 366.

[6] Nathan M., Dumke W., Burns G., Jr F. D., and Lasher G., "Stimulated Emission of Radiation from GaAs pn Junctions". Appl. Phys. Lett., 1962; 1: 62-64.

[7] Jr N. H., and Bevacqua S., "Coherent (visible) light emission from Ga (AsP) junctions". Appl. Phys. Lett., 1962; 1: 82.

[8] Akasaki I., Amano H., Itoh K., Koide N., and Manabe K., “GaN based UV/blue lightemitting devices, GaAs and Related Compounds conference". Inst. Phys. Conf. Ser., 1992; 127: 851.

[9] Nakamura S., Senoh M., Iwasa N., and Nagahama S., "High-brightness In-GaN blue, green and yellow light-emitting diodes with quantum well structures". Jpn. J. Appl. Phys., Part 2, 1995; 34:797.

[10] Muthu S., Schuurmans F. J. P., and Pashley M. D., "Red, Green, and Blue LEDs for White Light Illumination". IEEE J. Quantum Electron., 2002; 8: 333-338.

[11] Qasim Kh., Lei W., and Li Q., “Quantum Dots for Light Emitting Diodes”. J. Nanosci. Nanotechnol., 2013; 13: 3173-3185.

[12] Kim S., Im S. H., and Kim S.W., "Performance of light-emitting-diode based on quantum dots". Nanoscale, 2013; 5: 5205-5214.

[13] Sionnest P. G., "Electrical transport in colloidal quantum dot films". J. Phys. Chem. Lett., 2012; 3:1169-1175.

[14] Sun K., Vasudev M., Jung H.S., Yang J., Kar A., Li Y., Reinhardt P., Snee K., Stroscio M. A., and Dutta M., "Applications of colloidal quantum dots". Microelectron. J., 2009; 40: 644-649.

[15] Bae W. K., Park Y.S., Lim J., Lee D., Padilha L. A., McDaniel H., Robel I., Lee Ch., Pietryga J. M., and Klimov V. I., “Controlling the influence of Auger recombination 
on the performance of quantum-dot light-emitting diodes". Nat. Commun., 2013; 4: 1-8.

[16] Kumar B., Campbell S. A., and Ruden P. P., "Modeling charge transport in quantum dot light emitting devices with $\mathrm{NiO}$ and $\mathrm{ZnO}$ transport layers and Si quantum dots". J. Appl. Phys., 2013; 114: 044507.

[17] Kim J., Park Y. J., Kim Y., Kim Y.H., Han Ch. J., Han J. I., and Oh M. S., “Effects of Oxide Electron Transport Layer on Quantum Dots Light Emitting Diode with an Organic/Inorganic Hybrid Structure". Electron. Mater. Lett., 2013; 9: 779-782.

[18] Mashford B. S., Nguyen T. L., Wilsonb G. J., and Mulvaney P., “All-inorganic quantum-dot light-emitting devices formed via low-cost, wet-chemical processing". J. Mater. Chem., 2010; 20: 167-172.

[19] Amini E., Dolatyari M., Rostami A., Shekari H., Baghban H., Rasooli H., and Miri S., "Solution-Processed Photoconductive UV Detectors Based on ZnO Nanosheets". IEEE Photonics Technol. Lett., 2012; 24: 1995-1997.

[20] Willard D. M., Carillo L. L., Jung J., and Orden A. V., “CdSe-ZnS Quantum Dots as Resonance Energy Transfer Donors in a Model Protein-Protein Binding Assay". Nano Lett., 2001; 1: 469-474.

[21] Schubert E. F., "Light-Emitting Diodes". Cambridge University Press, Second edition 2006.

[22] Thorseth A., “Characterization, Modeling, and Optimization of Light-Emitting Diode Systems". Ph.D. Thesis, DTU Fotonic, 2011.

[23] Xi Y. Y., Hsu Y. F., Djurišić A. B., Ng A. M. C., Chan W. K., Tam H. L., and Cheah K. $\mathrm{W} .$, "NiO/ZnO light emitting diodes by solution-based growth". Appl. Phys. Lett., 2008; 92: 113505.

[24] Amini P., Rostami A., Dolatyari M., Rostami G., Torabi P., Mathur S., Singh T., "High performance Solution Processed Inorganic Quantum Dot LEDs" IEEE, J. Nanotech., 2014; submitted.

[25] Irwin M. D., Servaites J. D., Buchholz D. B., Leever B. J., Liu J., Emery J. D., Zhang M., Song J.H., Durstock M. F., Freeman A. J., Bedzyk M. J., Hersam M. C., Chang R. P. H., Ratner M. A., and Marks T. J., "Structural and Electrical Functionality of NiO Interfacial Films in Bulk Heterojunction Organic Solar Cells". Chem. Mater., 2011; 23: 2218-2226.

[26] L. Jang W., Lu Y. M., Hwang W.S., Hsiung T. L., and Wang H. P., "Point defects in sputtered NiO films". Appl. Phys. Lett., 2009; 94: 062103.

[27] Oh M. S., Hwang D.K., Seong D. J., Hwang H.S., Park S.J., and Kim E.D., "Improvement of Characteristics of Ga-Doped ZnO Grown by Pulsed Laser Deposition Using Plasma-Enhanced Oxygen Radicals". J. Electrochem. Soc., 2008; 155: D599-D603. 
[28] Gonc A. S. , alves, Davolos M. R., Masaki N., Yanagida S., Morandeira A., Durrant J. R., Freitasd J. N., and Nogueira A. F., "Synthesis and characterization of ZnO and ZnO:Ga films and their application in dye-sensitized solar cells". Dalton Trans., 2008; 1487-1491.

[29] Aneesh P. M., Vanaja K. A., and Jayaraj M. K., "Synthesis of ZnO nanoparticles by hydrothermal method". Nanophotonics Mater., 2007; 6639: 66390J1-9.

[30] Khan Z. R., Khan M. Sh., Zulfequar M., and Khan M. Sh., “Optical and Structural Properties of ZnO Thin Films Fabricated by Sol-Gel Method". Mater. Sci. Appl., 2011; 2: 340-345.

[31] Rao T.P., and Kumar M. C. S., "Resistivity Stability of Ga Doped ZnO Thin Films with Heat Treatment in Air and Oxygen Atmospheres". J. Cryst. Process Technol., 2012; 2: 72-79.

[32] Zheng J. H., Song J. L., Zhao Z., Jiang Q., and Lian J. S., “Optical and magnetic properties of Nd-doped ZnO nanoparticles". Cryst. Res. Technol., 2012; 1-6.

[33] Sahare P. D., and Kumar V., “Optical and Magnetic Properties of Cu-Doped ZnO Nanoparticles". International Journal of Innovative Technology and Exploring Engineering, 2013; 3: 16-21.

[34] Sapsford K. E., Berti L., and Medintz I. L., “Materials for Fluorescence Resonance Energy Transfer Analysis: Beyond Traditional Donor-Acceptor Combinations". Angew. Chem., Int. Ed., 2006; 45: 4562-4588.

[35] Nizamoglu S., Sari E., Baek J. H., Lee I. H., Sun X. W. and Demir H. V., “FRET-LEDs involving colloidal quantum dot nanophosphors". Journal of Light Emitting Diodes., 2010; 2: 1-5. 